\title{
Two-Dimensional Defective Crystals with Non-constant Dislocation Density and Unimodular Solvable Group Structure
}

\author{
Gareth Parry $^{1}$ (D) Maxim Zyskin ${ }^{1}$
}

Received: 30 November 2017 / Published online: 28 June 2018

(C) The Author(s) 2018

\begin{abstract}
In Parry and Zyskin (J. Elast. 127:249-268, 2017) we outlined mathematical methods which seemed to be necessary in order to discuss crystal structures with nonconstant dislocation density tensor (ddt). This was part of a programme to investigate the geometry of continuously defective crystals and the symmetries of associated discrete structures - one can think of the programme as an attempt to generalize the use of crystallographic groups as material symmetries in non-linear elasticity theory, for perfect crystals, to deal with the case where defects are present.

The methods used rely on the following fact: when the ddt is non-constant, (given technical assumptions), there is a Lie group that acts on the set of material points, and the dimension of the group is strictly greater than that of the ambient space in which the crystal resides. So there is a non-trivial isotropy group associated with the group action. We develop ideas, and recap the requisite mathematical apparatus, in the context of Davini's model of defective crystals, then focus on a particular case where the ddt is such that a solvable three dimensional Lie group acts on a two dimensional crystal state. We construct the corresponding discrete structures too.

The paper is an extension of Parry and Zyskin (J. Elast. 127:249-268, 2017), where the analogous group was nilpotent.
\end{abstract}

Keywords Crystals $\cdot$ Defects $\cdot$ Lie groups

Mathematics Subject Classification (2010) 74E20 · 74E25

\section{Introduction}

In this section we develop a connection between constitutive assumptions relating to the continuum mechanics of crystals and associated discrete structures. In the simplest case where the strain energy density depends only on point values of 'lattice vector fields' it

\footnotetext{
$凶$ G. Parry

gareth.parry@nottingham.ac.uk

1 School of Mathematical Sciences, University of Nottingham, Nottingham NG7 2RD, UK
} 
is commonly taken for granted that there is an associated discrete lattice (with the point values of the vector fields providing a basis of the lattice)—we argue that, if assumptions are formulated appropriately, then it is because the energy is presumed to depend only on the lattice vectors that the corresponding discrete structure is a lattice. In fact we give this argument in generality, in the next subsection, and comment on the simplest version of the argument subsequently.

Next, in this first section, we set up the restriction to two dimensional crystals, and indicate how Lie groups appear as a consequence of assumptions relating to the choice of kinematical variables in the strain energy density. We conclude the section by outlining the content of the paper and motivate the work by placing the geometrical issues in a mechanical context.

In Sect. 2 we recap the apparatus employed in [1], for convenience, then we move on to the extension of [1] to the case of solvable groups of a certain type.

\subsection{Basic Idea}

The simplest relevant constitutive assumption is that the continuum strain energy density, per unit current volume of a crystalline material, depends on three 'lattice vectors' $\boldsymbol{l}_{0}, \boldsymbol{l}_{1}, \boldsymbol{l}_{2} \in \mathbb{R}^{3}$, and traditionally the continuum so specified is locally associated with a perfect 'arithmetic' lattice $L$, where

$$
L \equiv\left\{\boldsymbol{x} \in \mathbb{R}^{3}: \boldsymbol{x}=n_{0} \boldsymbol{l}_{0}+n_{1} \boldsymbol{l}_{1}+n_{2} \boldsymbol{l}_{2}, n_{0}, n_{1}, n_{2} \in \mathbb{Z}\right\} .
$$

We explain how this particular constitutive assumption can lead to the lattice $L$, in a succession of steps.

(i) In Davini's continuum model of defective crystals [2], the primary kinematical objects are three 'lattice vector fields', $\boldsymbol{l}_{0}(\cdot), \boldsymbol{l}_{1}(\cdot), \boldsymbol{l}_{2}(\cdot)$, defined at all points $\boldsymbol{x}$ of a region $\Omega \subseteq \mathbb{R}^{3}$, so that the current geometric configuration of the material is, by definition, the crystal state

$$
\Sigma \equiv\left\{\boldsymbol{l}_{i}(\boldsymbol{x}): \boldsymbol{x} \in \Omega, i=0,1,2\right\} .
$$

With the simplest constitution assumption, the strain energy density at a point $\boldsymbol{x}_{0} \in \Omega$ is taken to have the form

$$
w=w\left(\boldsymbol{l}_{0}, \boldsymbol{l}_{1}, \boldsymbol{l}_{2}\right), \quad \boldsymbol{l}_{i} \equiv \boldsymbol{l}_{i}\left(\boldsymbol{x}_{0}\right), \quad i=0,1,2, \boldsymbol{x}_{0} \in \Omega,
$$

for some real valued function $w$. This energy density is to correspond in some way to the local structure of the material, close to $\boldsymbol{x}_{0}$, and it is an entirely reasonable prejudice (conditioned by the context - that the continuum represents a crystal, and so represents a collection of atoms, etc.) that both of the following assumptions hold:

- the local structure is discrete (i.e., there is a non-zero minimum separation between pairs of points in the structure);

- the structure has a symmetry determined by $\boldsymbol{l}_{0}, \boldsymbol{l}_{1}, \boldsymbol{l}_{2}$-in the traditional view, $\left\{\boldsymbol{l}_{0}, \boldsymbol{l}_{1}, \boldsymbol{l}_{2}\right\}$ is a basis of $L$, which is a discrete subgroup of $\mathbb{R}^{3}$ with addition as group operation, denoted $\left(\mathbb{R}^{3},+\right)$.

We contend that this second assumption can be seen, in fact, as a concomitant of the constitutive assumption (1.3), and explain why this is so in the next few subsections. 
(ii) Note that (1.3) is an analogue of the 'simple material' constitutive relation of non-linear elasticity, where energy density depends on deformation gradient only. Indeed, in the particular case where the crystal state is subject to an elastic deformation $\boldsymbol{u}: \Omega \rightarrow$ $\boldsymbol{u}(\Omega)$ so that the lattice vector fields $\boldsymbol{l}_{i}(\cdot)$ are changed to $\tilde{\boldsymbol{l}}_{i}(\cdot), i=0,1,2$, with

$$
\tilde{\boldsymbol{l}}_{i}(\boldsymbol{u}(\boldsymbol{x}))=\nabla \boldsymbol{u}(\boldsymbol{x}) \boldsymbol{l}_{i}(\boldsymbol{x}), \quad i=0,1,2, \boldsymbol{x} \in \Omega,
$$

and the crystal state $\Sigma$ is changed to $\tilde{\Sigma}$, with

$$
\tilde{\Sigma} \equiv\left\{\tilde{\boldsymbol{l}}_{i}(\boldsymbol{y}): \boldsymbol{y} \in \boldsymbol{u}(\Omega), i=0,1,2\right\},
$$

the strain energy density at the image point $\boldsymbol{y}_{0} \equiv \boldsymbol{u}\left(\boldsymbol{x}_{0}\right)$ is

$$
w\left(\tilde{\boldsymbol{l}}_{0}, \tilde{\boldsymbol{l}}_{1}, \tilde{\boldsymbol{l}}_{2}\right), \quad \text { where } \tilde{\boldsymbol{l}}_{i} \equiv \tilde{\boldsymbol{l}}_{i}\left(\boldsymbol{y}_{0}\right)=\nabla \boldsymbol{u}\left(\boldsymbol{x}_{0}\right) \boldsymbol{l}_{i},
$$

via (1.3), (1.4). Thus the strain energy density is determined by $\nabla \boldsymbol{u}\left(\boldsymbol{x}_{0}\right)$ if $\boldsymbol{l}_{0}, \boldsymbol{l}_{1}, \boldsymbol{l}_{2}$ are fixed (one may regard $l_{0}, l_{1}, l_{2}$ as defining a fixed reference configuration, in the class of purely elastic changes of state), and so (1.3) is a generalization of the simple material idea to this context.

(iii) Now it is important to realize that, if $\Sigma$ is given, there are many objects (calculated via the fields $\left.\boldsymbol{l}_{0}(\cdot), \boldsymbol{l}_{1}(\cdot), \boldsymbol{l}_{2}(\cdot)\right)$ which transform to corresponding objects in $\tilde{\Sigma}$, under elastic deformation, via transformation rules determined by the deformation gradient only (i.e. higher gradients of the deformation do not appear). For example, if $\boldsymbol{d}_{0}(\cdot), \boldsymbol{d}_{1}(\cdot), \boldsymbol{d}_{2}(\cdot)$ are the duals of the vector fields $\boldsymbol{l}_{0}(\cdot), \boldsymbol{l}_{1}(\cdot), \boldsymbol{l}_{2}(\cdot)$, and $n(\cdot) \equiv \boldsymbol{d}_{0}(\cdot)$. $\boldsymbol{d}_{1}(\cdot) \wedge \boldsymbol{d}_{2}(\cdot)>0$ (by assumption), then the dislocation density tensor $S(\cdot)=\left(S_{a b}(\cdot)\right)$, $a, b=0,1,2$, defined by

$$
S_{a b}(\cdot) \equiv \nabla \wedge \boldsymbol{d}_{a}(\cdot) \cdot \boldsymbol{d}_{b}(\cdot) / n(\cdot)
$$

transforms as follows,

$$
\tilde{S}_{a b}(\boldsymbol{u}(\boldsymbol{x}))=S_{a b}(\boldsymbol{x}), \quad a, b=0,1,2, \boldsymbol{x} \in \Omega,
$$

where $\tilde{S}(\cdot)$ is determined from $\tilde{\Sigma}$ via the analogue of (1.7). So if one incorporates a dependence on first order gradients of the lattice vector fields in (1.3), via the particular form

$$
w=w\left(\boldsymbol{l}_{0}, \boldsymbol{l}_{1}, \boldsymbol{l}_{2}, S\right),
$$

where $S \equiv S\left(x_{0}\right)$, and if $\Sigma$ is subject to elastic deformation $\boldsymbol{u}$, then the value of the strain energy density at the image point $\boldsymbol{y}_{0}=\boldsymbol{u}\left(\boldsymbol{x}_{0}\right)$ becomes

$$
w\left(\tilde{\boldsymbol{l}}_{0}, \tilde{\boldsymbol{l}}_{1}, \tilde{\boldsymbol{l}}_{2}, \tilde{S}\right)=w\left(\tilde{\boldsymbol{l}}_{0}, \tilde{\boldsymbol{l}}_{1}, \tilde{\boldsymbol{l}}_{2}, S\right)
$$

via (1.8), and this quantity is clearly determined by deformation gradient $\nabla \boldsymbol{u}\left(\boldsymbol{x}_{0}\right)$, if $\boldsymbol{l}_{0}, \boldsymbol{l}_{1}, \boldsymbol{l}_{2}, S$ are fixed. So (1.9) is also a generalization of the simple material idea to this context.

Objects which are unchanged by elastic deformation in the sense of (analogues of) (1.8) are called scalar elastic invariants, and there is an infinite number of such objects, depending on gradients of the lattice vector fields of arbitrary order (see Davini and 
Parry [3, 4], Olver [5], Parry and Silhavy [6], for more information). For example, $\boldsymbol{l}_{a} \cdot \nabla S$ is also a scalar elastic invariant, for each $a=0,1,2$, and so

$$
w=w\left(\left\{\boldsymbol{l}_{a}\right\}, S,\left\{\boldsymbol{l}_{a} \cdot \nabla S\right\}\right),
$$

where $\left\{\boldsymbol{l}_{a}\right\}$ denotes $\left\{\boldsymbol{l}_{0}, \boldsymbol{l}_{1}, \boldsymbol{l}_{2}\right\}$, etc., represents a generalization of the simple material idea too.

(iv) We need a couple of further observations before we can see how to 'interpret' the constitutive assumption (1.3) in a mathematical way, and deduce that we should associate an arithmetic lattice with it.

Let

$$
\boldsymbol{b}_{a}(\boldsymbol{x}) \equiv \nabla \wedge \boldsymbol{d}_{a}(\boldsymbol{x}), \quad \boldsymbol{x} \in \Omega, a=0,1,2
$$

denote the three 'Burgers vector' fields. Also define

$$
\boldsymbol{L}_{0}(\boldsymbol{x}) \equiv\left[\boldsymbol{l}_{1}(x), \boldsymbol{l}_{2}(\boldsymbol{x})\right] \equiv\left(\boldsymbol{l}_{2}(\boldsymbol{x}) \cdot \nabla\right) \boldsymbol{l}_{1}(\boldsymbol{x})-\left(\boldsymbol{l}_{1}(\boldsymbol{x}) \cdot \nabla\right) \boldsymbol{l}_{2}(\boldsymbol{x})
$$

with similar expressions for $\boldsymbol{L}_{1}(\boldsymbol{x}), \boldsymbol{L}_{2}(\boldsymbol{x})$, so that $\boldsymbol{L}_{0}(\cdot), \boldsymbol{L}_{1}(\cdot), \boldsymbol{L}_{2}(\cdot)$ are the three Lie brackets of pairs of lattice vector fields. (Note the sign convention in (1.13).) Recall that the flows generated by two vector fields commute if and only if the corresponding Lie bracket vanishes. Then one can show, [6], that

$$
S_{a b}(\boldsymbol{x})=\boldsymbol{b}_{a}(\boldsymbol{x}) \cdot \boldsymbol{d}_{b}(\boldsymbol{x}) / n(\boldsymbol{x})=\boldsymbol{L}_{b}(\boldsymbol{x}) \cdot \boldsymbol{d}_{a}(\boldsymbol{x}), \quad a, b=0,1,2, \boldsymbol{x} \in \Omega,
$$

so there is a simple relation between the Burgers vectors and the set of Lie brackets. From (1.13), (1.14) one may deduce that (dropping the point of evaluation)

$$
\boldsymbol{L}_{b}=S_{a b} \boldsymbol{l}_{a}, \quad\left[\boldsymbol{L}_{b}, \boldsymbol{l}_{c}\right]=S_{a c}\left[\boldsymbol{l}_{a}, \boldsymbol{l}_{b}\right]+\left(l_{b} \cdot \nabla S_{a c}\right) \boldsymbol{l}_{a}, \quad a, b, c=0,1,2,
$$

where summation convention applies. From (1.15), if $\boldsymbol{l}_{0}, \boldsymbol{l}_{1}, \boldsymbol{l}_{2}, S$ are known, so are $\boldsymbol{L}_{0}, \boldsymbol{L}_{1}, \boldsymbol{L}_{2}$ and one can replace $w\left(\boldsymbol{l}_{0}, \boldsymbol{l}_{1}, \boldsymbol{l}_{2}, S\right)$ by

$$
\bar{w}\left(\left\{\boldsymbol{l}_{a}\right\},\left\{\boldsymbol{L}_{a}\right\}\right),
$$

for some function $\bar{w}$, determined by $w$ and $(15)_{1}$. Similarly, if $\boldsymbol{l}_{0}, \boldsymbol{l}_{1}, \boldsymbol{l}_{2}, S,\left\{\boldsymbol{l}_{a} . \nabla S\right\}$ are known, then from $(15)_{1},(15)_{2}$ one can replace $w\left(\boldsymbol{l}_{0}, \boldsymbol{l}_{1}, \boldsymbol{l}_{2}, S,\left\{\boldsymbol{l}_{a} . \nabla S\right\}\right)$ by

$$
\tilde{w}\left(\left\{\boldsymbol{l}_{a}\right\},\left\{\boldsymbol{L}_{a}\right\},\left\{\left[\boldsymbol{L}_{a}, \boldsymbol{l}_{b}\right]\right\}\right) .
$$

Each of these constitutive forms, (1.16) and (1.17), is a generalization of the simple material idea. Let us consider the difference between (1.16) and (1.17) - the terms $\left\{\left[\boldsymbol{L}_{b}, \boldsymbol{l}_{c}\right]\right\}$ which appear in (1.17) are omitted in (1.16). We shall take this omission as a suggestion that the lattice vector fields which represent the local geometrical structure in (1.16) are such that the Lie brackets $\left\{\left[\boldsymbol{L}_{b}, \boldsymbol{l}_{c}\right]\right\}$ are determined in terms of those constitutive variables which do appear in (1.16), namely $\left\{\boldsymbol{l}_{a}\right\}$ and $\left\{\boldsymbol{L}_{a}\right\}$, and this is so if each $\boldsymbol{L}_{a}(\cdot), a=0,1,2$, is determined as a linear combination of $\boldsymbol{l}_{0}(\cdot), \boldsymbol{l}_{1}(\cdot), \boldsymbol{l}_{2}(\cdot)$. So we interpret (1.17) analogously, as a suggestion to consider crystal states where the lattice vector fields $\left[\boldsymbol{L}_{b}, \boldsymbol{l}_{c}\right](\cdot), b, c=0,1,2$, are given as linear combinations of $\left\{\boldsymbol{l}_{a}(\cdot)\right\}$ and $\left\{\boldsymbol{L}_{a}(\cdot)\right\}$.

In this paper, we shall just study this last suggestion, cf., (1.20) below, and so assume that $\left[\boldsymbol{L}_{b}, \boldsymbol{l}_{c}\right](\cdot), b, c=0,1,2$, are given as linear combinations of $\left\{\boldsymbol{l}_{a}(\cdot)\right\}$ and $\left\{\boldsymbol{L}_{a}(\cdot)\right\}$ 
throughout. This assumption has a clear kinematical interpretation: given the lattice vector fields $\left\{\boldsymbol{l}_{a}\right\}$ one constructs the corresponding Lie brackets $\left\{\boldsymbol{L}_{a}\right\}$ (or equivalently the Burgers vector fields), then one iterates the construction to produce $\left\{\left[\boldsymbol{L}_{a}, \boldsymbol{l}_{b}\right]\right\}$ (in the first instance) —one can continue this iteration indefinitely, but the assumption is that the vector fields so produced are all linear combinations of $\left\{\boldsymbol{l}_{a}\right\}$ and $\left\{\boldsymbol{L}_{a}\right\}$, so these two sets of fields provide a basis for the set of all vector fields generated in this way, in this case.

Mathematically: in case (1.16), the lattice vector fields $\boldsymbol{l}_{0}(\cdot), \boldsymbol{l}_{1}(\cdot), \boldsymbol{l}_{2}(\cdot)$ provide a basis for a three-dimensional Lie algebra of vector fields (we assume that the lattice vector fields are linearly independent at each $\boldsymbol{x} \in \Omega$ ); and in case (1.17) $\left\{\boldsymbol{l}_{a}(\cdot)\right\},\left\{\boldsymbol{L}_{a}(\cdot)\right\}$ include a basis for a $d$-dimensional Lie algebra of vector fields, $3<d \leq 6$. Elżanowski and Preston [7] call the Lie algebras that appear in this formalism 'lattice algebras' and we shall use that term here-a lattice algebra is a finite dimensional subalgebra of the algebra of smooth vector fields on $\Omega$, generated by the lattice vector fields. For case (1.3) we assume that the lattice algebra is 3-dimensional, with basis $\left\{\boldsymbol{l}_{a}(\cdot)\right\}$, and that the lattice vector fields are such that $\left\{\boldsymbol{L}_{a}(\boldsymbol{x})\right\}=\mathbf{0}, a=0,1,2, \boldsymbol{x} \in \Omega$.

(v) So, if we accept the suggestions made, for each form of energy density function ((1.3), (1.16), (1.17) or any higher order analogue thereof) there is a corresponding lattice algebra, and the whole apparatus of Lie group theory becomes available. For example, in case (1.3), the Lie brackets $\boldsymbol{L}_{i}(\cdot) \equiv 0, i=0,1,2$, the lattice vector fields commute, the lattice algebra is trivial and there is a corresponding abelian Lie group which one may take to be $\left(\mathbb{R}^{3},+\right)$. Finally, according to Bourbaki [8], the only discrete subgroups of $\left(\mathbb{R}^{3},+\right)$ are the arithmetic lattices, and we may associate the three-dimensional arithmetic lattice $L$ with basis $\left\{\boldsymbol{l}_{0}, \boldsymbol{l}_{1}, \boldsymbol{l}_{2}\right\}$ with the energy density (1.3), as anticipated.

(vi) In general, this approach gives a hierarchy of 'types' of defective crystal state. First, as noted in (v), if the Lie brackets are zero, the lattice vector fields commute with each other (pairwise), and the crystal state is an elastic deformation of a perfect crystal. Second, if the Lie brackets are non-zero, but are linearly dependent on the lattice vector fields, so that the dislocation density is constant, from (1.15), the crystal state can be represented as a Lie group. Last, if the Lie brackets are non-zero, not linearly dependent on the lattice vector fields (so that the dislocation density is not constant), but any 'lattice algebra' assumption holds, we shall see that there is a Lie group which acts on the crystal state in such a way that the lattice vector fields arise by projection from certain types of vector fields on the group (the Lie group has a higher dimension than that of the crystal configuration), see (2.41) below. One associates the constitutive relations (1.3), (1.16), (1.17) with the first, second and last type of defective crystal, respectively.

\subsection{Restriction to Two-Dimensional Crystal States}

In this paper we simplify the above setting a little by restricting attention to crystal states where two linearly independent vector fields are defined on a region $\Omega \subseteq \mathbb{R}^{2}$. Henceforward, then,

$$
\Sigma \equiv\left\{l_{i}(x): x \in \Omega \subseteq \mathbb{R}^{2}, i=0,1\right\} .
$$

Our interest is in the case where the dimension of the lattice algebra is strictly greater than the number of lattice vector fields, so we take the simplest option where the dimension is 3 . Thus the lattice algebra has basis

$$
\boldsymbol{l}_{0}(\cdot), \boldsymbol{l}_{1}(\cdot),\left[\boldsymbol{l}_{0}, \boldsymbol{l}_{1}\right](\cdot),
$$

and we are in two-dimensional analogue of (1.17) above. 
Accordingly, the assumption made in Sect. 1.1, point (iv), requires that the last two of the following equations hold:

$$
\begin{aligned}
& {\left[\boldsymbol{l}_{0}, \boldsymbol{l}_{1}\right](\cdot)=0 \boldsymbol{l}_{0}(\cdot)+0 \boldsymbol{l}_{1}(\cdot)+1\left[\boldsymbol{l}_{0}, \boldsymbol{l}_{1}\right](\cdot),} \\
& {\left[\boldsymbol{l}_{0},\left[\boldsymbol{l}_{0}, \boldsymbol{l}_{1}\right]\right](\cdot)=\alpha \boldsymbol{l}_{0}(\cdot)+\beta \boldsymbol{l}_{1}(\cdot)+\gamma\left[\boldsymbol{l}_{0}, \boldsymbol{l}_{1}\right](\cdot),} \\
& {\left[\boldsymbol{l}_{1},\left[\boldsymbol{l}_{0}, \boldsymbol{l}_{1}\right]\right](\cdot)=\bar{\alpha} \boldsymbol{l}_{0}(\cdot)+\bar{\beta} \boldsymbol{l}_{1}(\cdot)+\bar{\gamma}\left[\boldsymbol{l}_{0}, \boldsymbol{l}_{1}\right](\cdot),}
\end{aligned}
$$

$\alpha, \beta, \gamma, \bar{\alpha}, \bar{\beta}, \bar{\gamma} \in \mathbb{R}$. Equations (1.20) define the lattice algebra, and the constants which appear are the 'structure constants'. There is an isomorphism class of connected and simply connected Lie groups with Lie algebra isomorphic to that so defined, so we shall be interested, below, in the relation between the vector fields $\boldsymbol{l}_{0}(\cdot), \boldsymbol{l}_{1}(\cdot)$ (and $\left[\boldsymbol{l}_{0}, \boldsymbol{l}_{1}\right](\cdot)$ ) and some three-dimensional Lie group, $G$ say.

Now from [7], Olver [9], Palais [10], we may assume (given (1.20)) that $G$ has a Lie algebra $\mathfrak{g}$ with basis $\boldsymbol{v}_{0}, \boldsymbol{v}_{1}, \boldsymbol{v}_{2}$ such that

$$
\begin{aligned}
& {\left[\boldsymbol{v}_{0}, \boldsymbol{v}_{1}\right]=0 \boldsymbol{v}_{0}+0 \boldsymbol{v}_{1}+1 \boldsymbol{v}_{2},} \\
& {\left[\boldsymbol{v}_{0}, \boldsymbol{v}_{2}\right]=\alpha \boldsymbol{v}_{0}+\beta \boldsymbol{v}_{1}+\gamma \boldsymbol{v}_{2},} \\
& {\left[\boldsymbol{v}_{1}, \boldsymbol{v}_{2}\right]=\bar{\alpha} \boldsymbol{v}_{0}+\bar{\beta} \boldsymbol{v}_{1}+\bar{\gamma} \boldsymbol{v}_{2},}
\end{aligned}
$$

(same structure constants as (1.20)), and that there exists a group action $\lambda: G \times \Omega \rightarrow \Omega$ (with properties given in Sect. 2) such that

$$
\begin{aligned}
& \nabla_{1} \lambda(\mathbf{0}, \boldsymbol{x}) \boldsymbol{v}_{a}=\boldsymbol{l}_{a}(\boldsymbol{x}), \quad a=0,1, \\
& \nabla_{1} \lambda(\mathbf{0}, \boldsymbol{x}) \boldsymbol{v}_{3}=\left[\boldsymbol{l}_{0}, \boldsymbol{l}_{1}\right](\boldsymbol{x}),
\end{aligned}
$$

$\boldsymbol{x} \in \Omega$, where $\nabla_{1} \lambda(\mathbf{0}, \boldsymbol{x})$ denotes the gradient of $\lambda$ with respect to its first argument, and $\mathbf{0}$ denotes the group identity. $\nabla_{1} \lambda(\mathbf{0}, \boldsymbol{x})$ maps $\mathfrak{g}$ into the set of vector fields on $M$, and provides a Lie algebra homomorphism (see Sect. 2.3 below). Knowing $G, \lambda,\left\{\boldsymbol{v}_{i}\right\}$ gives us the lattice vector fields, via (1.22), and with this interpretation, the lattice vector fields are infinitesimal generators corresponding to the group action.

\section{Remarks}

- The fact that $\left[\boldsymbol{l}_{0}, \boldsymbol{l}_{1}\right](\cdot)$ is not a linear combination of $\boldsymbol{l}_{0}(\cdot)$ and $\boldsymbol{l}_{1}(\cdot)$ implies that the dislocation density tensor is non-constant in $\Omega$.

- Three-dimensional Lie groups and algebras have been extensively studied and classified, for example, by Bianchi [11] and Jacobsen [12] - this amounts to a classification of structure constants modulo change of basis. According to [12] there are four different classes: the abelian, nilpotent, solvable and simple classes of Lie algebras. The abelian case leads to traditional crystallography, and we considered the nilpotent case in [1]. The solvable case divides into unimodular and non-unimodular classes-we consider the unimodular case here, and hope to present the non-unimodular solvable and (so-called) simple case in forthcoming works.

- Let $\mathfrak{g}_{2}$ denote the algebra generated by Lie algebra elements of the form $[\boldsymbol{g}, \boldsymbol{h}]$, $\boldsymbol{g}, \boldsymbol{h} \in \mathfrak{g}$, and call it the derived algebra (or commutator algebra, or commutator ideal as $\left[\mathfrak{g}, \mathfrak{g}_{2}\right]=\boldsymbol{g}_{2}$ ). Define $\mathfrak{g}^{1} \equiv \mathfrak{g}, \mathfrak{g}^{2}=\left[\mathfrak{g}, \mathfrak{g}^{1}\right], \mathfrak{g}^{3}=\left[\mathfrak{g}, \mathfrak{g}^{2}\right] \ldots \mathfrak{g}^{n}=\left[\mathfrak{g}, \mathfrak{g}^{n-1}\right]$. Then $\mathfrak{g}$ is nilpotent if $\mathfrak{g}^{n}=\{\mathbf{0}\}$ for some $n \in \mathbb{Z}$. Also define $\mathfrak{g}_{1}=\mathfrak{g}, \mathfrak{g}_{2}=\left[\mathfrak{g}_{1}, \mathfrak{g}_{1}\right] \ldots \mathfrak{g}_{n}=$ 
$\left[\mathfrak{g}_{n-1}, \mathfrak{g}_{n-1}\right]$. Then $\mathfrak{g}$ is solvable if $\mathfrak{g}_{n}=\{\mathbf{0}\}$ for some $n \in \mathbb{Z}$. It will turn out, in the solvable case that we consider here, where $\operatorname{dim} \mathfrak{g}=3$, that $\left[\mathfrak{g}_{2}, \mathfrak{g}_{2}\right]=\{\mathbf{0}\}$, so that the commutators of algebra elements commute with each other, and this simplifying factor facilitates many of the computations below.

- In the case that $\operatorname{dim} \mathfrak{g}=3, \mathfrak{g}$ is simple if and only if $\operatorname{dim} \mathfrak{g}_{2}=3$.

- We shall construct canonical forms of the bracket relations, (1.20) or (1.21), explicitly, as many have done before, in cases where $\operatorname{dim} \mathfrak{g}_{2} \neq 3$, arriving at Jacobsen's results, but follow a route which is a little different to Jacobsen's, just to illustrate the method.

\subsection{Outline of Content}

We consider two-dimensional crystal states (1.18) and three-dimensional lattice algebras with basis (1.19). The task will be to identify, from amongst certain canonical forms of the lattice algebras and corresponding Lie groups, those which generate discrete structures along the lines indicated in Sect. 1.1. More precisely, we shall presume that the structures are produced by a definite iteration procedure-a starting point $\boldsymbol{x}_{0} \in \Omega$ is presumed given, and others are generated by discrete flow along the integral lines of the 'primary' lattice vector fields $\boldsymbol{l}_{0}(\cdot)$ and $\boldsymbol{l}_{1}(\cdot)$. It turns out that this procedure leads to the study of a subgroup of $G$ generated by two group elements-we denote the two generators by $a_{0}$ and $a_{1}$, and the subgroup by $D\left(a_{0}, a_{1}\right)$. There is a projection mapping $\pi: G \rightarrow \Omega$, determined by the group action, which is such that $\pi\left(D\left(a_{0}, a_{1}\right)\right)$ is the structure produced by the iterative procedure above, and we study whether or not this structure is discrete. The contents of the paper are briefly as follows.

In Sect. 2 we recall various facts and definitions from the theory of Lie groups and homogeneous spaces. We introduce the algebra of right invariant fields on $G$ and note that each such field is determined by its value at the group identity. We discuss group actions on a manifold briefly and note (as intimated in the previous section) that there is a Lie algebra homomorphism from the algebra of right invariant fields on $G$ to the lattice algebra. We show how to construct the projection mapping $\pi: G \rightarrow \Omega$ mentioned.

In Sect. 3 we focus on solvable Lie groups and algebras. We provide canonical forms of the algebras, to start, arriving at results in [12]. We use an ansatz to deduce one form of group composition function and then modify this via elastic deformation/group isomorphism to write the group composition as a semi-direct product which also has a matrix representation (in terms of $4 \times 4$ matrices). This matrix representation is used in Anslander, Green and Hahn [13], and we recall and reformulate a couple of results from [13]. This work, [13], gives a list of discrete subgroups of $G$ with compact fundamental domain, modulo group isomorphism - such subgroups have three generators (recall that we are interested in subgroups with two generators).

In Sect. 4 we derive canonical forms of the lattice vector fields $\boldsymbol{l}_{0}(\cdot), \boldsymbol{l}_{1}(\cdot)$ (and $\left[\boldsymbol{l}_{0}, \boldsymbol{l}_{1}\right](\cdot)$ ), for unimodular solvable groups. We do this in two ways-one way is a direct analytical method which uses the bracket relations derived in Sect. 3 and assumed analyticity properties of the vector fields, the other way uses homogeneous space ideas. It seems to us that the latter method is a more algorithmic, simpler, process.

Finally, in Sect. 5, we calculate $\pi\left(D\left(a_{0}, a_{1}\right)\right)$ in each case, and determine whether or not this set is discrete.

\subsection{Mechanical Context and Motivation}

The constitutive functions, in this model of defective crystals, depend only on the point values of the lattice vector fields and certain of their directional derivatives, so that (from the 
continuum mechanics perspective) the 'current' set of vector fields determines the mechanical properties of the material-a priori there is no 'reference' or 'intermediate' configuration, and energy density, stress, etc., are to be determined by the point values of the current fields. With technical assumptions, these fields are enough to determine corresponding discrete structures (obtained by discrete flow along the lattice vector fields). For example, in the perfect crystal case, one obtains a perfect crystal lattice in this way, as described in Sect. 1.1(v) — this lattice has certain symmetries (because different choices of basis in (1.1) give the same lattice), in particular the crystallographic point groups are the orthogonal symmetries of the lattice.

Now, traditionally, in variational problems which purport to describe the mechanics of perfect crystals, the crystallographic point groups are taken as material symmetry groups of the corresponding energy density, and the set of competitor functions generally corresponds to elastic deformation of the crystal state. This procedure (if it is to be used with Davini's model) requires one definition, and one fact: first one needs to know what is meant by elastic deformation of a crystal state defined by the lattice vector fields - it is usually stated that the lattice vector fields are embedded in the elastic deformation (which means that (1.4) above holds), or that the 'Cauchy-Born hypothesis' holds. Second one needs to know that the point group transformations, which map the discrete perfect lattice to itself, can be extended to a mapping of the continuum, i.e., that the crystallographic group transformations can be extended to elastic deformations (in fact one also needs to know that this extension is unique). Note that it is easy to verify this fact in this simple, perfect crystal, case.

This paper is part of a programme of work to set out the geometry of defective crystal states in low dimensions (dimensions 2 or 3), to study the corresponding discrete structures and their symmetries, to investigate whether or not these symmetries extend to 'material symmetries' of the continuum, and to consider corresponding variational problems. In effect, the geometric part of this amounts to the study of vector fields (or frames) on low dimensional manifolds - a great deal is known about this, of course.

The connection with the theory of Lie groups was outlined in Sect. 1.1(iv), and this allows us to discuss defective crystals of a certain 'type' mathematically. Viewed from a Lie group perspective, with the assumptions of Sect. 1.1(iv), the crystal state (that is, the distribution of lattice vector fields on the body manifold) is obtained as the projection of a distribution of well-defined fields on the Lie group (the group manifold is generally of higher dimension than the body manifold). The discrete structures, obtained as above, become projections of discrete subgroups of the continuous Lie group, and the question regarding whether or not the 'symmetries' of those structures extend in a certain way becomes a question of group theory. In fact, since the crystal state is obtained from a higher dimension Lie group via some projection, we are concerned with the theory of homogeneous spaces rather than Lie groups per se. It may be a surprise to the reader that the 'one fact' above, needed to extend point group symmetries to elastic deformation in the perfect crystal case, does not hold in full generality, though it is true in the various cases that we have considered explicitly so far. (It might be, then, that the Cauchy Born hypothesis cannot hold in principle, for sufficiently complex defective materials.)

We are concerned here just with one part of the programme, the derivation of discrete structures corresponding to particular three dimensional Lie groups acting on two dimensional continua. Cases where the dimension of the Lie group and the body manifold dimension are equal $(=3)$, have been considered previously. We consider here neither the symmetries of the discrete structures, nor any related variational problems. However we note that issues relating to the formulation of variational problems, and particularly the selection of the competitor functions which strictly include the elastic deformations, are considered in $[3,4]$, and that corresponding rigorous calculations are given in Fonseca and Parry [14]. 


\section{Lie Groups and Homogeneous Spaces}

\subsection{Lie Groups and Algebras}

Let $G$ be a Lie group, so that $G$ has the structure of a manifold and the group multiplication and inverse maps are smooth. We consider three-dimensional Lie groups, so a group element is uniquely determined by three real numbers $x_{0}, x_{1}, x_{2}$, called the coordinates of the group element. We identify the group element with its coordinates and write

$$
\boldsymbol{x} \equiv\left(\begin{array}{c}
x_{0} \\
x_{1} \\
x_{2}
\end{array}\right) \in G
$$

We may suppose that the coordinate system is such that the group identity has coordinates $0,0,0$, so we can say that

$$
\mathbf{0} \equiv\left(\begin{array}{l}
0 \\
0 \\
0
\end{array}\right) \in G
$$

represents the group identity, as we did in the previous section. Let $\boldsymbol{x}^{-1} \in G$ denote the inverse of $x \in G$. Let $\boldsymbol{\psi}: G \times G \rightarrow G$ denote the group composition function, so that the coordinates of $\boldsymbol{\psi}(\boldsymbol{x}, \boldsymbol{y}) \in G$ are those of the product of $\boldsymbol{x} \in G$ and $\boldsymbol{y} \in G$. Then

$$
\psi(\mathbf{0}, \boldsymbol{x})=\psi(x, 0)=x, \quad \psi\left(x^{-1}, x\right)=\psi\left(x, x^{-1}\right)=0 .
$$

Also the group product is associative, so

$$
\psi(x, \psi(y, z))=\psi(\psi(x, y), z), \quad x, y, z \in G .
$$

Let

$$
\left.A_{i j k} \equiv \frac{\partial^{2} \psi_{i}}{\partial x_{j} \partial y_{k}}(\boldsymbol{x}, \boldsymbol{y})\right|_{\boldsymbol{x}=\boldsymbol{y}=\mathbf{0}}, \quad i, j, k=0,1,2,
$$

and let

$$
C_{i j k} \equiv A_{i j k}-A_{i k j}=\varepsilon_{s j k} \varepsilon_{s p q} A_{i p q},
$$

when $\varepsilon_{i j k}$ are the components of the permutation symbol. The constants $C_{i j k}$ are called the structure constants of the Lie algebra which corresponds to $G$, with respect to the given choice of coordinates. The associativity of the group product implies that the structure constants satisfy the Jacobi identity, namely that

$$
C_{i j k} C_{j r s}+C_{i j r} C_{j s k}+C_{i j s} C_{j k r}=0 .
$$

In general (that is, without reference to the above), a Lie algebra $\mathfrak{g}$ is a vector space together with a bilinear, skew-symmetric 'bracket' product $[\cdot, \cdot]: \mathfrak{g} \times \mathfrak{g} \rightarrow \mathfrak{g}$ which satisfies the identity,

$$
[\boldsymbol{X},[\boldsymbol{Y}, \boldsymbol{Z}]]+[\boldsymbol{Y},[\boldsymbol{Z}, \boldsymbol{X}]]+[\boldsymbol{Z},[\boldsymbol{X}, \boldsymbol{Y}]]=\mathbf{0}, \quad \boldsymbol{X}, \boldsymbol{Y}, \boldsymbol{Z} \in \mathfrak{g} .
$$

Note that if the vector space is $\mathbb{R}^{3}$ and one sets

$$
[\boldsymbol{X}, \boldsymbol{Y}]=C_{i j k} X_{j} Y_{k} \boldsymbol{e}_{i}
$$


where $\left\{\boldsymbol{e}_{0}, \boldsymbol{e}_{1}, \boldsymbol{e}_{2}\right\}$ is a basis of $\mathbb{R}^{3}$, and where the constants $C_{i j k}=-C_{i k j}$ satisfy (2.7), then this particular bracket product is evidently bilinear and skew-symmetric, and it satisfies (2.8) (by virtue of (2.7)).

\subsection{Right Invariant Fields}

If one fixes $y \in G$, then the function $\psi(\cdot, y): G \rightarrow G$ represents multiplication on the right by $\boldsymbol{y}$, and the gradient of this function will be denoted $\nabla_{1} \boldsymbol{\psi}(\cdot, \boldsymbol{y})$. A vector field $\boldsymbol{v}$ is an object defined at each $\boldsymbol{x} \in G$ taking values in the tangent space to the group manifold at $\boldsymbol{x}$. The tangents to the three coordinate curves in the group manifold provide a basis for the tangent space at a given $\boldsymbol{x} \in G$ and we write the components of $\boldsymbol{v}$ as $\left(\begin{array}{l}v_{0}(x) \\ v_{1}(x) \\ v_{2}(x)\end{array}\right)$ with respect to this basis, at this point. if

The vector field $\boldsymbol{v}$ is said to be right invariant with respect to the composition function $\psi$

$$
v(\psi(x, y))=\nabla_{1} \psi(x, y) v(x), \quad x, y \in G .
$$

Given a vector field $\boldsymbol{v}$, the integral curve $\boldsymbol{x}(t)$ passing through initial point $\boldsymbol{x}_{0}$, associated with $\boldsymbol{v}$, is the solution of the ordinary differential equation

$$
\frac{d x}{d t}(t)=v(x(t)), \quad x(0)=x_{0} .
$$

It can be shown that the integral curve corresponding to a right invariant vector field is complete, in the sense that (2.11) has a solution well defined for all real values $t$. So, for each $t \in \mathbb{R}$, one can define a mapping $\exp (t \boldsymbol{v}): G \rightarrow G$ by

$$
\exp (t \boldsymbol{v})\left(\boldsymbol{x}_{0}\right)=\boldsymbol{x}(t), \quad \boldsymbol{x} \in U,
$$

where $\boldsymbol{x}(t)$ is the integral curve passing through initial point $\boldsymbol{x}_{0}$, associated with (the right invariant vector field) $v$. One also defines the point $e^{t v} \in G$ by

$$
e^{t v} \equiv \exp (t \boldsymbol{v})(\mathbf{0})
$$

It is standard that

$$
\exp (t \boldsymbol{v})(\boldsymbol{x})=\boldsymbol{\psi}\left(e^{t \boldsymbol{v}}, \boldsymbol{x}\right)
$$

so that the flow corresponding to the mapping $\exp (t \boldsymbol{v})$ is simply related to the integral curve which passes through the origin.

The points $\{\boldsymbol{x}(t), t \in \mathbb{R}\}$ which make up the integral curve of a right invariant field $\boldsymbol{v}$, passing through the origin, are the elements of a one parameter subgroup of $G$, so that

$$
\psi(x(t), x(s))=x(t+s), \quad t, s \in \mathbb{R},
$$

and the converse is also true (see Pontryagin [15], for example). This one parameter subgroup may be written as $e^{\langle\boldsymbol{v}\rangle}$, where $\langle\boldsymbol{v}\rangle=\{t \boldsymbol{v} ; t \in \mathbb{R}\}$ is the span of $\boldsymbol{v}$, and $\boldsymbol{v} \equiv \boldsymbol{v}(\mathbf{0})$.

The set of right invariant fields is a vector space with respect to pointwise addition and real scalar multiplication. Indeed note that by differentiating (2.4) with respect to $x$ and then putting $\boldsymbol{x}=\mathbf{0}$,

$$
\nabla_{1} \boldsymbol{\psi}(\mathbf{0}, \boldsymbol{\psi}(\boldsymbol{y}, \boldsymbol{z}))=\nabla_{1} \boldsymbol{\psi}(\boldsymbol{y}, \boldsymbol{z}) \nabla_{1} \boldsymbol{\psi}(\mathbf{0}, \boldsymbol{y})
$$


Then by comparing (2.16) and (2.10) one sees that the three vector fields whose components are given by

$$
\boldsymbol{l}_{a}(\boldsymbol{y}) \equiv\left(\begin{array}{l}
\frac{\partial \psi_{0}}{\partial x_{a}}(\mathbf{0}, \boldsymbol{y}) \\
\frac{\partial \psi_{1}}{\partial x_{a}}(\mathbf{0}, \boldsymbol{y}) \\
\frac{\partial \psi_{2}}{\partial x_{a}}(\mathbf{0}, \boldsymbol{y})
\end{array}\right), \quad a=0,1,2,
$$

are right invariant, and one may show that these three fields together form a basis for the set of all right invariant fields. Also, by putting $\boldsymbol{x}=\mathbf{0}$ in (2.10), one notes that any right invariant field is determined by its value at the group identity. Thus for any right invariant field $\boldsymbol{v}(\cdot)$ with components $v_{i}(\cdot), i=0,1,2$, we have

$$
v_{i}(\boldsymbol{y})=\frac{\partial \psi_{i}}{\partial x_{a}}(\mathbf{0}, \boldsymbol{y}) v_{a}(\mathbf{0}) .
$$

\subsection{Lie Algebras of Right Invariant Fields}

Let $\boldsymbol{l}(\cdot), \boldsymbol{m}(\cdot)$ be right invariant fields with components $l_{i}(\cdot), m_{i}(\cdot), i=0,1,2$ respectively. Then from (2.18),

$$
l_{i}(\boldsymbol{y})=\frac{\partial \psi_{i}}{\partial x_{a}}(\mathbf{0}, \boldsymbol{y}) \boldsymbol{l}_{a}(\mathbf{0}), \quad m_{i}(\boldsymbol{y})=\frac{\partial \psi_{i}}{\partial x_{a}}(\mathbf{0}, \boldsymbol{y}) m_{a}(\mathbf{0})
$$

One calculates that the $i$ th component of $\{(\boldsymbol{m} . \nabla) \boldsymbol{l}-(\boldsymbol{l} . \nabla) \boldsymbol{m}\}(\boldsymbol{y})$ is

$$
m_{b}(\boldsymbol{y}) \frac{\partial^{2} \psi_{i}}{\partial x_{a} \partial y_{b}}(\mathbf{0}, \boldsymbol{y}) l_{a}(0)-l_{b}(\boldsymbol{y}) \frac{\partial^{2} \psi_{i}}{\partial x_{a} \partial y_{b}}(\mathbf{0}, \boldsymbol{y}) m_{a}(\mathbf{0}),
$$

so that from (2.5) and (2.6), the $i$ th component of

$$
\{(\boldsymbol{m} \cdot \nabla) \boldsymbol{l}-(\boldsymbol{l} \cdot \nabla) \boldsymbol{m}\}(\mathbf{0})
$$

is

$$
A_{i j k} l_{j}(\mathbf{0}) m_{k}(\mathbf{0})-A_{i k j} l_{j}(\mathbf{0}) m_{k}(\mathbf{0}) \equiv C_{i j k} l_{j}(\mathbf{0}) m_{k}(\mathbf{0}) .
$$

Bearing (2.9) and (2.21) in mind, define the Lie bracket of vector fields (not necessarily right invariant vector fields) by

$$
[\boldsymbol{l}(\cdot), \boldsymbol{m}(\cdot)]=\{(\boldsymbol{m} \cdot \nabla) \boldsymbol{l}-(\boldsymbol{l} \cdot \nabla) \boldsymbol{m}\}(\cdot),
$$

again noting the sign convention. For right invariant fields $\boldsymbol{l}(\cdot)$ and $\boldsymbol{m}(\cdot)$, we have from (2.21) that the $i$ th component of the Lie bracket is

$$
[\boldsymbol{l}(\mathbf{0}), \boldsymbol{m}(\mathbf{0})]_{i}=C_{i j k} l_{j}(\mathbf{0}) m_{k}(\mathbf{0}), \quad i=0,1,2 .
$$

Putting $\boldsymbol{l}(\mathbf{0}) \equiv \boldsymbol{l}, \boldsymbol{m}(\mathbf{0})=\boldsymbol{m}, l_{i}(\mathbf{0}) \equiv l_{i}, m_{i}(\mathbf{0}) \equiv m_{i}$, this gives

$$
[\boldsymbol{l}, \boldsymbol{m}]_{i}=C_{i j k} l_{j} m_{k} .
$$

Comparing with (2.9), we see that the set of right invariant vector fields, with this choice of Lie bracket, gives a Lie algebra. (One can show that the Lie bracket of right invariant 
fields is right invariant.) Thus one can think of the Lie algebra of $G$ either as the vector space $\mathbb{R}^{3}$, with Lie bracket (2.9), or as the vector space of right invariant fields with Lie bracket (2.22). We shall denote the Lie algebra of $G$ by $\mathfrak{g}$, not distinguishing between these two interpretations.

\subsection{Group and Algebra Homomorphisms}

Let $\mathfrak{g}$ and $\mathfrak{h}$ be Lie algebras with Lie brackets $[\cdot, \cdot]_{\mathfrak{g}},[\cdot, \cdot]_{\mathfrak{h}}$ respectively. (In the context of this paper, both brackets $[\cdot, \cdot]_{\mathfrak{g}},[\cdot, \cdot]_{\mathfrak{h}}$ map $\mathbb{R}^{3} \times \mathbb{R}^{3} \rightarrow \mathbb{R}^{3}$.) A Lie algebra homomorphism is a linear transformation $L: \mathfrak{g} \rightarrow \mathfrak{h}$ which satisfies

$$
[L \boldsymbol{x}, L \boldsymbol{y}]_{\mathfrak{h}}=L[\boldsymbol{x}, \boldsymbol{y}]_{\mathfrak{g}}, \quad \boldsymbol{x}, \boldsymbol{y} \in \mathfrak{g} .
$$

If $C_{i j k}^{\mathfrak{g}}, C_{i j k}^{\mathfrak{h}}$ are the structure constants for $\mathfrak{g}, \mathfrak{h}$ with respect to bases $\left\{\boldsymbol{e}_{i}\right\},\left\{\boldsymbol{f}_{i}\right\}$ respectively, then

$$
C_{i j k}^{\mathfrak{h}} L_{j p} L_{k q}=L_{i r} C_{r p q}^{\mathfrak{g}},
$$

where $L \boldsymbol{e}_{i}=L_{j i} \boldsymbol{f}_{j}, i, j=0,1,2$.

Let $G$ and $H$ be Lie groups with group multiplication functions $\psi_{G}, \psi_{H}$ respectively. A smooth mapping $\phi: G \rightarrow H$ is a Lie group homomorphism if

$$
\psi_{H}(\phi(x), \phi(y))=\phi\left(\psi_{G}(x, y)\right), \quad x, y \in G .
$$

If $\mathfrak{g}$ is the Lie algebra of $G$, and $\mathfrak{h}$ is the Lie algebra of $H$, and $\phi: G \rightarrow H$ is a Lie group homomorphism, then $\nabla \boldsymbol{\phi}(\mathbf{0}) \equiv L$ is a Lie algebra homomorphism. Conversely if $L$ satisfies (2.25), then there exists a Lie group homomorphism $\boldsymbol{\phi}$ such that $\nabla \boldsymbol{\phi}(\mathbf{0})=L$. Also,

$$
\boldsymbol{\phi}\left(e^{\boldsymbol{v}}\right)=e^{(\nabla \boldsymbol{\phi}(\mathbf{0}) \boldsymbol{v})}, \quad \boldsymbol{v} \in \mathfrak{g} \equiv \mathbb{R}^{3},
$$

where $\phi$ satisfies (2.27), where the exponential on the left hand side of (2.28) is the exponential which maps $\mathfrak{g}$ to $G$, and that on the right hand side maps $\mathfrak{h}$ to $H$. Relation (2.28) allows one to calculate the Lie group homomorphisms explicitly if the Lie algebra homomorphisms are found by solving (2.26). $\phi: G \rightarrow G$ (resp. $L: \mathfrak{g} \rightarrow \mathfrak{g}$ ) is called an automorphism. $\left(\phi(\cdot)\right.$ and $\phi^{-1}(\cdot)$ have to be smooth.)

Now suppose that, in (2.26), $L$ is invertible, so that the dimensions of $\mathfrak{g}$ and $\mathfrak{h}$ are necessarily the same, and suppose that the structure constants in $\mathfrak{g}$ are known. We can use (2.26) to find the structure constants in $\mathfrak{h}$ :

$$
C_{i p q}^{(\mathfrak{h})}=L_{i r} C_{r j k}^{(\mathfrak{g})} L_{j p}^{-1} L_{k q}^{-1} .
$$

For three-dimensional Lie algebras, define

$$
T_{i m}^{(\mathfrak{h})}=C_{i p q}^{(\mathfrak{h})} \varepsilon_{m p q},
$$

where $\varepsilon_{m p q}$ is the permutation symbol, so that

$$
T_{i m}^{(\mathfrak{h})}=L_{i r} C_{r j k}^{(\mathfrak{g})}\left(\varepsilon_{m p q} L_{j p}^{-1} L_{k q}^{-1}\right) .
$$

Since

$$
L_{s m}^{-1}\left(\varepsilon_{m p q} L_{j p}^{-1} L_{k q}^{-1}\right)=\frac{1}{\operatorname{det} L} \varepsilon_{m p q},
$$


we find that

$$
T_{i m}^{(\mathfrak{h})}=L_{i r} C_{r j k}^{(\mathfrak{g})}\left(\frac{1}{\operatorname{det} L} \varepsilon_{s j k} L_{m s}\right)=\frac{1}{\operatorname{det} L} L_{i r} T_{r s}^{(\mathfrak{g})} L_{m s} .
$$

Thus

$$
T^{(\mathfrak{h})}=\frac{1}{\operatorname{det} L} L T^{(\mathfrak{g})} L^{T}
$$

Later, we shall use (2.33) to transform the structure constants to some simple canonical forms. Later, too, we shall also use the automorphisms of $\mathfrak{h}$, namely those invertible transformations $L$ which satisfy

$$
T^{(\mathfrak{h})}=\frac{1}{\operatorname{det} L} L T^{(\mathfrak{h})} L^{T}
$$

to simplify further calculations.

\subsection{Left Action of a Group on a Manifold}

Let $\lambda: G \times M \rightarrow M$ denote a left action of the Lie group $G$ on the manifold $M$, so that for $m \in M, \boldsymbol{g}_{1}, \boldsymbol{g}_{2} \in G$,

$$
\lambda(\mathbf{0}, m)=m, \quad \lambda\left(\boldsymbol{g}_{1}, \lambda\left(\boldsymbol{g}_{2}, m\right)\right)=\lambda\left(\boldsymbol{\psi}\left(\boldsymbol{g}_{1}, \boldsymbol{g}_{2}\right), m\right) .
$$

The infinitesimal generators of the group action are defined as

$$
\boldsymbol{l}_{\boldsymbol{v}}(m)=\nabla_{1} \lambda(\mathbf{0}, m) \boldsymbol{v}, \quad \boldsymbol{v} \in \mathfrak{g}, m \in M .
$$

Note particularly that $\nabla_{1} \lambda(\mathbf{0}, m)$ maps elements of $\mathfrak{g}$ to vector fields on $M$, and that it can be shown that this mapping is a Lie algebra homomorphism (from $\mathfrak{g}$ to the lattice algebra). Thus

$$
\nabla_{1} \lambda(\mathbf{0}, m)[\boldsymbol{v}, \boldsymbol{w}]=\left[\nabla_{1} \lambda(\mathbf{0}, m) \boldsymbol{v}, \nabla_{1} \lambda(\mathbf{0}, m) \boldsymbol{w}\right], \quad \boldsymbol{v}, \boldsymbol{w} \in \mathfrak{g}, m \in M .
$$

Also we have the following result (anticipated in Sect. 1): let $\boldsymbol{w}_{0}(\cdot), \boldsymbol{w}_{1}(\cdot), \boldsymbol{w}_{2}(\cdot)$ be vector fields on $M$ such that $\left[\boldsymbol{w}_{i}, \boldsymbol{w}_{j}\right](\cdot)=C_{k i j} w_{k}(\cdot), i, j, k=0,1,2$, for constant $C_{k i j}=-C_{k j i}$ satisfying the Jacobi identity (2.7). Then there exists a Lie group $G$, corresponding Lie algebra $\mathfrak{g}$ with (the same) structure constants $C_{i j k}$ relative to some basis $\boldsymbol{v}_{0}, \boldsymbol{v}_{1}, \boldsymbol{v}_{2}$ of $\mathfrak{g}$, and a (local) group action $\lambda: G \times M \rightarrow M$ such that for $m \in M$,

$$
\boldsymbol{w}_{i}(m)=\nabla_{1} \lambda(\mathbf{0}, m) \boldsymbol{v}_{i}, \quad i=0,1,2
$$

see $[7,9,10]$.

Finally, in this subsection, by differentiating $(2.35)_{2}$ with respect to $\boldsymbol{g}_{1}$, setting $\boldsymbol{g}_{1}=\mathbf{0}$ and $\boldsymbol{g}_{2}=\boldsymbol{g} \in G$ we get

$$
\nabla_{1} \lambda(\mathbf{0}, \lambda(\boldsymbol{g}, m))=\nabla_{1} \lambda(\boldsymbol{g}, m) \nabla_{1} \boldsymbol{\psi}(\mathbf{0}, \boldsymbol{g})
$$

so if $\boldsymbol{v} \in \mathfrak{g}$,

$$
\nabla_{1} \lambda(\mathbf{0}, \lambda(\boldsymbol{g}, m)) \boldsymbol{v}=\nabla_{1} \lambda(\boldsymbol{g}, m)\left\{\nabla_{1} \boldsymbol{\psi}(\mathbf{0}, \boldsymbol{g}) \boldsymbol{v}\right\}
$$


The term in brackets in (2.40) is the right invariant field $\boldsymbol{v}(\cdot)$, with $\boldsymbol{v}(\boldsymbol{0})=\boldsymbol{v}$, evaluated at $\boldsymbol{g} \in G$, and the left side of (2.40) is an infinitesimal generator of the group action, recalling (2.36). So for $\boldsymbol{v} \in \mathfrak{g}$,

$$
\boldsymbol{l}_{\boldsymbol{v}}(\boldsymbol{\lambda}(\boldsymbol{g}, m))=\nabla_{1} \boldsymbol{\lambda}(\mathbf{0}, m) \boldsymbol{v}(\boldsymbol{g}),
$$

and this relation connects the right invariant field $\boldsymbol{v}(\cdot)$ with the infinitesimal generator $\boldsymbol{l}_{v}(\cdot)$, $\boldsymbol{v}=\boldsymbol{v}(\mathbf{0})$. Note that $\nabla_{1} \lambda(\cdot, \cdot)$ is singular in the case that the dimensions of $M$ and $\mathfrak{g}$ are different.

\subsection{Homogeneous Space}

Define the isotropy group of the left action $\lambda: G \times M \rightarrow M$ by

$$
I_{m}=\{g \in G: \lambda(g, m)=m\},
$$

for any $m \in M$, and suppose that the action is transitive, so

$$
\lambda(G, m)=M
$$

for any $m \in M$. Then $(G, M)$ is called a homogeneous space.

Let $H$ be a subgroup of $G$ and define the left coset space $G / H$ by

$$
G / H=\{\boldsymbol{k} H ; \boldsymbol{k} \in G\} .
$$

Note that if two group elements are juxtaposed, one should understand group composition, i.e., if $\boldsymbol{a}, \boldsymbol{b} \in G, \boldsymbol{a} \boldsymbol{b} \equiv \psi(\boldsymbol{a}, \boldsymbol{b})$, so $\boldsymbol{k} H \equiv\{\boldsymbol{\psi}(\boldsymbol{k}, \boldsymbol{h}) ; \boldsymbol{h} \in H\}$, in particular.

According to Komrakov [16], we have the following results:

- If $H$ is a closed subgroup of $G$, then $G / H$ can be given the structure of a manifold, with

$\lambda: G \times G / H \rightarrow G / H$ defined by $\lambda(\boldsymbol{g}, \boldsymbol{k} H)=\boldsymbol{\psi}(\boldsymbol{g}, \boldsymbol{k}) H$ smooth and transitive. Then $(G, G / H)$ is a homogeneous space.

- If $(G, M)$ is a homogeneous space, and $m \in M$, then $I_{m}$ is a closed subgroup of $G$.

So, given a transitive group action $\lambda$, any isotropy group is a closed subgroup of $G$, and given any closed subgroup of $G$ one can construct a corresponding group action. In fact, define the projection mapping $\pi: G \rightarrow G / H$ by $\pi(g)=g H$ and choose a section $\sigma: G / H \rightarrow G$ such that $\boldsymbol{\pi}(\boldsymbol{\sigma}(\boldsymbol{g} H))=\boldsymbol{g} H$. (Note that it is not generally true that there exists a well defined global section, but that we verify the existence of such a section in each case of interest below.) Then $\lambda$ can be expressed as:

$$
\lambda(\boldsymbol{g}, \boldsymbol{k} H)=\pi(\psi(\boldsymbol{g}, \boldsymbol{\sigma}(\boldsymbol{k} H)))
$$

and this shows how to construct the group action from any closed subgroup. (Note that $\lambda$, defined by (2.45), is independent of the choice of section.) One can also show that

$$
H=I_{\pi(\mathbf{0})},
$$

if $\lambda$ is so expressed, so any closed subgroup is an isotropy group. 


\subsection{Flow Along Infinitesimal Generators}

Recall that infinitesimal generators of the group action are defined, for any $\boldsymbol{v} \in \mathfrak{g}$, by

$$
\boldsymbol{l}_{\boldsymbol{v}}(m)=\nabla_{1} \lambda(\mathbf{0}, m) \boldsymbol{v}, \quad m \in M .
$$

The flow along these vector fields corresponds, by definition, to the solution $\{m(\varepsilon) ; \varepsilon \in \mathbb{R}\}$ of

$$
\frac{d}{d \varepsilon} m(\varepsilon)=\boldsymbol{l}_{\boldsymbol{v}}(m(\varepsilon)), \quad m(0)=\lambda(\boldsymbol{g}, m)
$$

given $g \in G, m \in M$. It can be shown, as in [1], that

$$
m(\varepsilon)=\lambda\left(e^{\varepsilon v} \boldsymbol{g}, m\right),
$$

so that the flow along the infinitesimal generators, or lattice vector fields, in $\Omega$, corresponds to multiplication by elements of a one parameter subgroup of $G$, modulo the group action. (Also recall that, in (2.49), $e^{\varepsilon v} \boldsymbol{g}$ means $\boldsymbol{\psi}\left(e^{\varepsilon v}, \boldsymbol{g}\right)$.)

\section{Solvable Groups and Algebras}

First we investigate three-dimensional solvable Lie algebras $\mathfrak{g}$ with derived algebra $\mathfrak{g}_{2}$ of dimension 1 or 2 . Then we construct the corresponding groups and reformulate results of [13] which list the (three generator) discrete subgroups (with compact fundamental domain) of the 'canonical' three dimensional Lie groups.

\section{$3.1 \operatorname{dim} \mathfrak{g}_{2}=1$}

Since $\mathfrak{g}_{2}$ is one-dimensional we can assume it has basis $\boldsymbol{e}_{1}$, so

$$
\left.T^{(\mathfrak{g})}=\left(\begin{array}{lll}
0 & 0 & 0 \\
\alpha & \beta & \gamma \\
0 & 0 & 0
\end{array}\right), \quad \alpha^{2}+\beta^{2}+\gamma^{2}>0 . \quad \text { (We take } \boldsymbol{e}_{0}, \boldsymbol{e}_{1}, \boldsymbol{e}_{2} \text { as a basis of } \mathfrak{g} .\right)
$$

If $\alpha=\gamma=0, \beta \neq 0$, take $L=\operatorname{diag}(1,2 / \beta, 1)$ in (2.33) to get

$$
T^{(\mathfrak{h})}=\left(\begin{array}{lll}
0 & 0 & 0 \\
0 & 2 & 0 \\
0 & 0 & 0
\end{array}\right) .
$$

Since from (2.30)

$$
2 C_{i r s}^{(\mathfrak{h})}=T_{i m}^{(\mathfrak{h})} \varepsilon_{m r s},
$$

such $T^{(\mathfrak{h})}$ corresponds to a Lie algebra with basis $\boldsymbol{u}, \boldsymbol{v}, \boldsymbol{w}$ satisfying the commutation relations

$$
[u, v]=w, \quad[u, w]=[v, w]=0 .
$$


This defines the three-dimensional nilpotent Lie algebra considered in [1], so we do not consider this case any further here. So assume that not both of $\alpha$ and $\gamma$ are zero in (3.1). Since permutation matrices are allowed (for $L$ ) in (2.33) we can assume that $\gamma \neq 0$. Take

$$
L=\left(\begin{array}{ccc}
x_{3} & 0 & x_{0} \\
0 & 1 & x_{1} \\
0 & 0 & x_{2}
\end{array}\right), \quad x_{2} x_{3} \neq 0,
$$

and calculate that

$$
T^{(\mathfrak{h})}=\frac{1}{\operatorname{det} L} L T^{(\mathfrak{g})} L^{T}=\frac{1}{x_{2} x_{3}}\left(\begin{array}{ccc}
0 & 0 & 0 \\
x_{3} \alpha+x_{0} \gamma & \beta+x_{1} \gamma & x_{2} \gamma \\
0 & 0 & 0
\end{array}\right) .
$$

By choosing $x_{3}=\frac{1}{2} \gamma, x_{0}=-\frac{1}{2} \alpha, x_{1}=-\beta / \gamma, x_{2}=2 / \gamma$, we get

$$
T^{(\mathfrak{h})}=\left(\begin{array}{lll}
0 & 0 & 0 \\
0 & 0 & 2 \\
0 & 0 & 0
\end{array}\right) \equiv T
$$

Such $T^{(\mathfrak{h})} \equiv T$ corresponds to a solvable Lie algebra with basis $\boldsymbol{u}, \boldsymbol{v}, \boldsymbol{w}$ satisfying

$$
[u, v]=v, \quad[u, w]=[v, w]=0 .
$$

Note that the Jacobi identity is satisfied, when (3.7) holds.

Now consider the automorphisms of the algebra, when $T$ has the form (3.6). From (2.34) we get

$$
\left(\begin{array}{lll}
0 & 0 & 0 \\
0 & 0 & 2 \\
0 & 0 & 0
\end{array}\right)=\frac{2}{\operatorname{det} L}\left(\begin{array}{lll}
0 & 0 & L_{01} \\
0 & 0 & L_{11} \\
0 & 0 & L_{21}
\end{array}\right)\left(\begin{array}{ccc}
* & * & * \\
* & * & * \\
L_{02} & L_{12} & L_{22}
\end{array}\right),
$$

where $*$ denotes a number that plays no role in the calculation. Since $L$ is non-singular, the first and last row conditions imply that $L_{01}=L_{21}=0$. The second row condition implies that $L_{11} \neq 0, L_{02}=L_{12}=0, L_{11} L_{22} / \operatorname{det} L=1$, thus $L_{00}=1$. Therefore

$$
L=\left(\begin{array}{ccc}
1 & 0 & 0 \\
L_{10} & L_{11} & 0 \\
L_{20} & 0 & L_{22}
\end{array}\right), \quad L_{11} L_{22} \neq 0
$$

\section{Remarks}

- If $\boldsymbol{h}=s_{i} \boldsymbol{f}_{i}$ is a non-zero element of the Lie algebra (3.7), with $\boldsymbol{u}=\boldsymbol{f}_{0}, \boldsymbol{v}=\boldsymbol{f}_{1}, \boldsymbol{w} \equiv \boldsymbol{f}_{2}$, and if $L$ is an automorphism of the form (3.9), then

$$
L \boldsymbol{h}=\left(L_{i j} s_{j}\right) \boldsymbol{f}_{i} \equiv t_{i} \boldsymbol{f}_{i},
$$

where

$$
t_{0}=s_{0}, \quad t_{1}=L_{10} s_{0}+L_{11} s_{1}, \quad t_{2}=L_{20} s_{0}+L_{22} s_{2} .
$$

Then if $s_{0} \neq 0$ we can choose $L_{10}, L_{20}$ so that $t_{1}=t_{2}=0$, so that $\langle\boldsymbol{h}\rangle$ is mapped by an algebra automorphism to $\left\langle\boldsymbol{f}_{0}\right\rangle$. If $s_{0}=0$, we can choose $L_{11}, L_{22}$ to rescale coordinates, and bring $\langle\boldsymbol{h}\rangle$ to one of the forms $\left\langle\boldsymbol{f}_{1}\right\rangle,\left\langle\boldsymbol{f}_{2}\right\rangle,\left\langle\boldsymbol{f}_{1}+\boldsymbol{f}_{2}\right\rangle$. 
- If one replaces $\boldsymbol{w}$ by $\tilde{\boldsymbol{w}}=\boldsymbol{w}+\boldsymbol{v}$ in (3.7), the commutation relations become

$$
[u, v]=v, \quad[u, \bar{w}]=v, \quad[v, \bar{w}]=0,
$$

and, with this choice of basis, these relations have the form (1.21).

\section{$3.2 \operatorname{dim} \mathfrak{g}_{2}=2$}

Here we can assume that $\mathfrak{g}_{2}$ is spanned by $\boldsymbol{e}_{1}$ and $\boldsymbol{e}_{2}$, so $T^{(\mathfrak{g})}$ is of the form

$$
T^{(\mathfrak{g})}=\left(\begin{array}{ccc}
0 & 0 & 0 \\
a_{1} & Q \\
a_{2} & Q
\end{array}\right)
$$

where $Q$ is a $2 \times 2$ matrix. Under a particular transformation of the form

$$
L=\left(\begin{array}{ccc}
1 & 0 & 0 \\
0 & M \\
0 & M
\end{array}\right)
$$

where $M$ is a non-singular $2 \times 2$ matrix, $T^{(\mathfrak{g})}$ transforms as follows, via (2.33):

$$
T^{(\mathfrak{h})}=\left(\begin{array}{ccc}
0 & 0 & 0 \\
\frac{M a}{\operatorname{det} M} & \tilde{Q}
\end{array}\right), \quad \tilde{Q} \equiv \frac{M Q M^{\top}}{\operatorname{det} M}, \quad \boldsymbol{a} \equiv\left(\begin{array}{l}
a_{1} \\
a_{2}
\end{array}\right) .
$$

We shall show that $\boldsymbol{a}=\mathbf{0}$, by contradiction. So suppose $\boldsymbol{a} \neq 0$ and choose $M$ such that $\frac{M a}{\operatorname{det} M}=\left(\begin{array}{l}2 \\ 0\end{array}\right)$ to get

$$
T^{(\mathfrak{h})}=2\left(\begin{array}{ccc}
0 & 0 & 0 \\
1 & q_{11} & q_{12} \\
0 & q_{21} & q_{22}
\end{array}\right) .
$$

This corresponds to the commutation relations

$$
\begin{aligned}
& {\left[\boldsymbol{f}_{1}, \boldsymbol{f}_{2}\right]=\boldsymbol{f}_{1},} \\
& {\left[\boldsymbol{f}_{2}, \boldsymbol{f}_{0}\right]=q_{11} \boldsymbol{f}_{1}+q_{21} \boldsymbol{f}_{2},} \\
& {\left[\boldsymbol{f}_{0}, \boldsymbol{f}_{1}\right]=q_{12} \boldsymbol{f}_{1}+q_{22} \boldsymbol{f}_{2} .}
\end{aligned}
$$

However from the Jacobi identity we obtain $q_{22} \boldsymbol{f}_{2}+q_{21} \boldsymbol{f}_{1}=\mathbf{0}$, which implies that $q_{22}=$ $q_{21}=0$. But this implies that $\operatorname{dim} \mathfrak{g}_{2}=1$, contrary to supposition. So $\boldsymbol{a}=\mathbf{0}$ if $\operatorname{dim} \mathfrak{g}_{2}=2$.

Changing notation a little, according to (3.19) below, we can therefore assume that

$$
\begin{aligned}
& {\left[\boldsymbol{f}_{1}, \boldsymbol{f}_{2}\right]=0} \\
& {\left[\boldsymbol{f}_{0}, \boldsymbol{f}_{1}\right]=\sigma_{11} \boldsymbol{f}_{1}+\sigma_{12} \boldsymbol{f}_{2}} \\
& {\left[\boldsymbol{f}_{0}, \boldsymbol{f}_{2}\right]=\sigma_{21} \boldsymbol{f}_{1}+\sigma_{22} \boldsymbol{f}_{2}}
\end{aligned}
$$

where the $2 \times 2$ matrix $\sigma=\left(\sigma_{i j}\right)$ is such that, with $Q \equiv\left(q_{i j}\right)$,

$$
Q=\sigma^{\top} J, \quad J=\left(\begin{array}{cc}
0 & 1 \\
-1 & 0
\end{array}\right), J^{2}=-\mathbb{1},
$$


where $\mathbb{1}$ is the $2 \times 2$ identity matrix. (There should be no confusion between the matrix $\sigma$ and the section map $\sigma$ introduced in (2.45).)

Note that $Q$ and $\sigma$ must be non-singular, in order that $\operatorname{dim} \mathfrak{g}_{2}=2$. One calculates from the transformation rule for $Q$, which is $(15)_{2}$, when $L$ has the form (3.14), that

$$
\tilde{\sigma}^{\top}=-\frac{1}{\operatorname{det} M} M \sigma^{\top} J M^{\top} J
$$

which gives that

$$
\tilde{\sigma}^{\top}=M \sigma^{\top} M^{-1}
$$

since

$$
-\frac{1}{\operatorname{det} M} J M^{\top} J=M^{-1},
$$

for arbitrary non-singular $2 \times 2$ matrices $M$. So one can use similarity transformations to bring $\sigma$ to one of the standard canonical forms over the real numbers. One can also transform $\sigma^{\top}$ to $\sigma^{\top} / \mu$, for any $\mu \neq 0$, by rescaling $\boldsymbol{f}_{0}$ in (3.18), and it can be shown that there is no further flexibility gained by taking the most general non-singular matrix $L$ in (2.33). Thus $\sigma^{\top}$ may be replaced by

$$
\tilde{\sigma}^{\top}=\frac{1}{\mu} M \sigma^{\top} M^{-1}
$$

with $\mu \neq 0, M$ non-singular but otherwise arbitrary, i.e., $\sigma$ may be simplified (in (3.18)) by similarity transformations and overall scaling in what follows (cf. [12]).

Solvable Lie algebras of the form (3.18) are said to be unimodular if $\operatorname{tr} \sigma=0$, and we consider only this case in this paper (because it turns out that $\operatorname{tr} \sigma=0$ is a necessary condition for the existence of certain types of discrete subgroups, see the comments preceding (3.53) below). Now we can enumerate the various canonical forms of $\sigma$ :

(i) if $\sigma$ is diagonalizable with real eigenvalues, then by similarity and overall scaling one can assume that $\sigma=\operatorname{diag}(1, \lambda), \lambda \neq 0$. Given that the algebra is unimodular, $\lambda=-1$. Hence $\sigma=\operatorname{diag}(1,-1)$.

(ii) if $\sigma$ is not diagonalizable it can be put in the form $\left(\begin{array}{ll}\lambda & 1 \\ 0 & \lambda\end{array}\right), \lambda \neq 0$. No such matrix is unimodular.

(iii) if $\sigma$ has complex conjugate eigenvalues, it can be put in the form $\left(\begin{array}{cc}\lambda & \mu \\ -\mu & \lambda\end{array}\right), \lambda^{2}+\mu^{2} \neq 0$. Unimodularity gives that $\lambda=0$, and by overall scaling one can then assume that $\mu=1$. Hence

$$
\sigma=\left(\begin{array}{cc}
0 & 1 \\
-1 & 0
\end{array}\right) .
$$

Therefore there are two canonical forms of $\sigma$ to be considered here:

$$
\sigma=\left(\begin{array}{cc}
1 & 0 \\
0 & -1
\end{array}\right),\left(\begin{array}{cc}
0 & 1 \\
-1 & 0
\end{array}\right) .
$$

We calculate the automorphisms of the canonical Lie algebras, in the case that $T^{(\mathfrak{g})}=T$ has the form

$$
T=\left(\begin{array}{ccc}
0 & 0 & 0 \\
0 & Q \\
0 & Q
\end{array}\right)
$$


(recall (3.13), and recall that $\boldsymbol{a}=0$ if $\operatorname{dim} \mathfrak{g}_{2}=2$ ). Set

$$
L=\left(\begin{array}{ll}
L_{00} & \boldsymbol{w}^{\top} \\
L_{10} & M \\
L_{20} & M
\end{array}\right), \quad \boldsymbol{w}=\left(\begin{array}{c}
L_{01} \\
L_{02}
\end{array}\right)
$$

and impose condition (2.34) with $T^{(\mathfrak{h})}$ replaced by $T$ to get

$$
\left(\begin{array}{ccc}
0 & 0 & 0 \\
0 & Q \\
0 & Q
\end{array}\right)=\frac{1}{\operatorname{det} L}\left(\begin{array}{cc}
\boldsymbol{w}^{\top} Q \boldsymbol{w} & w^{\top} Q M^{\top} \\
M Q \boldsymbol{w} & M Q M^{\top}
\end{array}\right) .
$$

Since $Q$ is non-singular this implies $\boldsymbol{w}=\mathbf{0}, \operatorname{det} L=L_{00} \operatorname{det} M \neq 0$ and $(\operatorname{det} L) Q=$ $M Q M^{\top}$. Using (3.19) and (3.22) we get

$$
L_{00} \sigma^{\top} M=M \sigma^{\top} .
$$

Taking the determinant of both sides in (3.28), we have

$$
L_{00}= \pm 1
$$

(iv) Suppose that $\sigma=\left(\begin{array}{cc}1 & 0 \\ 0 & -1\end{array}\right)$ and $L_{00}=+1$, then one finds from (3.28) that $M$ is diagonal. If $L_{00}=-1$, one finds $M=\left(\begin{array}{cc}0 & \alpha \\ -\beta & 0\end{array}\right), \alpha \beta \neq 0$. Thus $L$ has the form

$$
\begin{aligned}
& \left(\begin{array}{lll}
1 & 0 & 0 \\
* & \alpha & 0 \\
* & 0 & \beta
\end{array}\right) \text { or }\left(\begin{array}{ccc}
-1 & 0 & 0 \\
* & 0 & \alpha \\
* & -\beta & 0
\end{array}\right)=\left(\begin{array}{ccc}
1 & 0 & 0 \\
* & \alpha & 0 \\
* & 0 & \beta
\end{array}\right)\left(\begin{array}{ccc}
-1 & 0 & 0 \\
0 & 0 & 1 \\
0 & -1 & 0
\end{array}\right), \\
& \alpha \beta \neq 0,
\end{aligned}
$$

(v) If $\sigma=\left(\begin{array}{cc}0 & 1 \\ -1 & 0\end{array}\right)$ one calculates similarly that $L$ has the form

$$
\begin{aligned}
& \left(\begin{array}{ccc}
1 & 0 & 0 \\
* & \alpha & \beta \\
* & -\beta & \alpha
\end{array}\right) \text { or }\left(\begin{array}{ccc}
-1 & 0 & 0 \\
* & \beta & \alpha \\
* & \alpha & -\beta
\end{array}\right)=\left(\begin{array}{ccc}
1 & 0 & 0 \\
* & \alpha & \beta \\
* & -\beta & \alpha
\end{array}\right)\left(\begin{array}{ccc}
-1 & 0 & 0 \\
0 & 0 & 1 \\
0 & 1 & 0
\end{array}\right) \\
& \alpha^{2}+\beta^{2} \neq 0,
\end{aligned}
$$

(cf., Ha and Lee [17]).

\section{Remarks}

- For later use, note that if $\boldsymbol{h}=s_{i} \boldsymbol{f}_{i}$ is a non-zero element of the Lie algebra with $\sigma=$ $\left(\begin{array}{cc}1 & 0 \\ 0 & -1\end{array}\right)$, and $L$ has the form (3.30), then $L \boldsymbol{h}=t_{i} \boldsymbol{f}_{i}$ where

$$
t_{0}=s_{0}, \quad t_{1}=\alpha s_{1}+* s_{0}, \quad t_{2}=\beta s_{2}+* s_{0},
$$

or

$$
t_{0}=-s_{0}, \quad t_{1}=\alpha s_{2}+* s_{0}, \quad t_{2}=-\beta s_{1}+* s_{0},
$$


where $\alpha \beta \neq 0$. Hence $\langle\boldsymbol{h}\rangle$ coincides with the span of one of the following choices of $\left(s_{0}, s_{1}, s_{2}\right)^{\top}$ :

$$
\left(\begin{array}{l}
1 \\
0 \\
0
\end{array}\right),\left(\begin{array}{l}
0 \\
1 \\
0
\end{array}\right),\left(\begin{array}{l}
0 \\
1 \\
1
\end{array}\right) .
$$

- If $\sigma=\left(\begin{array}{cc}0 & 1 \\ -1 & 0\end{array}\right)$, then similarly

$$
t_{0}=s_{0}, \quad t_{1}=\alpha s_{2}+\beta s_{2}+* s_{0}, \quad t_{2}=-\beta s_{1}+\alpha s_{2}+* s_{0},
$$

or

$$
t_{0}=-s_{0}, \quad t_{1}=\beta s_{1}+\alpha s_{2}+* s_{0}, \quad t_{2}=\alpha s_{1}-\beta s_{2}+* s_{0} .
$$

So $\langle\boldsymbol{h}\rangle$ coincides with the span of one of the following choices of $\left(s_{0}, s_{1}, s_{2}\right)^{\top}$ :

$$
\left(\begin{array}{l}
1 \\
0 \\
0
\end{array}\right),\left(\begin{array}{l}
0 \\
1 \\
0
\end{array}\right)
$$

(because $\left(\begin{array}{cc}\alpha & \beta \\ -\beta & \alpha\end{array}\right)\left(\begin{array}{l}s_{1} \\ s_{2}\end{array}\right)=\left(\begin{array}{l}1 \\ 0\end{array}\right)$ has a solution for $\left(\begin{array}{cc}\alpha & \beta \\ -\beta & \alpha\end{array}\right)$ with $\alpha \beta \neq 0$, given $\left.\left(\begin{array}{l}s_{1} \\ s_{2}\end{array}\right) \neq \mathbf{0}\right)$.

- If $\sigma=\left(\begin{array}{cc}1 & 0 \\ 0 & -1\end{array}\right)$, the corresponding commutation relations have the form $[\boldsymbol{u}, \boldsymbol{v}]=\boldsymbol{v}$, $[\boldsymbol{u}, \boldsymbol{w}]=-\boldsymbol{w},[\boldsymbol{v}, \boldsymbol{w}]=\mathbf{0}$. They may be put in the form of (1.21) by the change of basis $\boldsymbol{v} \rightarrow \tilde{\boldsymbol{v}} \equiv \boldsymbol{v}+\boldsymbol{w}, \boldsymbol{w} \rightarrow \tilde{\boldsymbol{w}}=\boldsymbol{v}-\boldsymbol{w}$. The commutation relations in the case $\sigma=\left(\begin{array}{cc}0 & 1 \\ -1 & 0\end{array}\right)$ are in the form (1.21).

\subsection{Group Multiplication}

First we derive a particular Lie group multiplication such that the commutation relations for a corresponding Lie algebra of vector fields are (3.18). This multiplication is (3.45) below, given without proof in [13] - it is easy enough to check that (3.45) leads to the required Lie algebra, but we could not find an a priori proof anywhere, so we provide one.

To begin with we identify particular vector fields which satisfy those relations using an ansatz. Then we construct a corresponding group composition function by solving the right invariance condition (2.10) for $\boldsymbol{\psi}$, given these vector fields. Finally we construct an isomorphic group composition function by using (2.27), with an appropriate smooth mapping, to obtain (3.45). This composition function represents a semi-direct product $\left(\mathbb{R}\right.$ acts on $\mathbb{R}^{2}$ ), and there is a matrix representation of this product, (3.47), which facilitates later computations.

So note that if vector fields $\boldsymbol{l}_{0}(\cdot), \boldsymbol{l}_{1}(\cdot), \boldsymbol{l}_{2}(\cdot)$ are defined by

$$
\boldsymbol{l}_{0}=(1,0,0), \quad \boldsymbol{l}_{1}=\left(0, l_{11}, l_{12}\right), \quad \boldsymbol{l}_{3}=\left(0, l_{21}, l_{22}\right),
$$

where $\boldsymbol{l}_{i}=\boldsymbol{l}_{i}\left(x_{0}\right), i=0,1,2$, where

$$
\left(\begin{array}{ll}
l_{11} & l_{12} \\
l_{21} & l_{22}
\end{array}\right)=e^{-\sigma x_{0}},
$$


where the exponential on the right side of (3.35) is the matrix exponential, then

$$
\frac{d}{d x_{0}}\left(\begin{array}{ll}
l_{11} & l_{12} \\
l_{21} & l_{22}
\end{array}\right)=-\sigma\left(\begin{array}{ll}
l_{11} & l_{12} \\
l_{21} & l_{22}
\end{array}\right) .
$$

One calculates that the vector fields satisfy

$$
\left[\boldsymbol{l}_{1}, \boldsymbol{l}_{2}\right]=0, \quad\left[\boldsymbol{l}_{0}, \boldsymbol{l}_{\alpha}\right]=\sigma_{\alpha \beta} \boldsymbol{l}_{\beta}, \quad \alpha, \beta=1,2,
$$

so that (3.18) holds, with $\boldsymbol{l}_{a}=f_{\boldsymbol{a}}, a=0,1,2$. Next from an analogue of (2.10), viz.

$$
\boldsymbol{l}_{a}\left(\boldsymbol{\psi}^{\prime}(\boldsymbol{s}, \boldsymbol{t})\right)=\nabla_{1} \boldsymbol{\psi}^{\prime}(\boldsymbol{s}, \boldsymbol{t}) \boldsymbol{l}_{a}(\boldsymbol{s}), \quad a=0,1,2,
$$

we find that (with $\left(\psi_{0}^{\prime}, \psi_{1}^{\prime}, \psi_{2}^{\prime}\right)$ the components of $\left.\psi^{\prime}\right)$ :

$$
\frac{\partial \psi_{0}^{\prime}}{\partial s_{0}}(\boldsymbol{s}, \boldsymbol{t})=1, \quad \frac{\partial \psi_{0}^{\prime}}{\partial s_{\alpha}}(\boldsymbol{s}, \boldsymbol{t})=0, \quad \alpha=1,2 .
$$

These equations give that $\psi_{0}^{\prime}(\boldsymbol{s}, \boldsymbol{t})=s_{0}+f(\boldsymbol{t})$, for some $f: \mathbb{R}^{3} \rightarrow \mathbb{R}$. Since $\boldsymbol{\psi}^{\prime}(\mathbf{0}, \boldsymbol{t})=\boldsymbol{t}$ we get that $\psi_{0}^{\prime}(\boldsymbol{s}, \boldsymbol{t})=s_{0}+t_{0}$. Using this fact, (3.38) also implies that

$$
\frac{\partial \psi_{\alpha}^{\prime}}{\partial s_{0}}(\boldsymbol{s}, \boldsymbol{t})=0, \quad \frac{\partial \psi_{\alpha}^{\prime}}{\partial s_{\beta}}(\boldsymbol{s}, \boldsymbol{t})=\left(e^{-t_{0} \sigma}\right)_{\alpha \beta}^{\top}, \quad \alpha, \beta=1,2 .
$$

Now we amend notation a little and write

$$
\boldsymbol{s}=\left(\begin{array}{c}
s_{0} \\
s_{1} \\
s_{2}
\end{array}\right) \quad \text { as } \quad\left(s_{0},\left(\begin{array}{c}
s_{1} \\
s_{2}
\end{array}\right)\right) \equiv\left(s_{0}, s\right), \quad s_{0} \in \mathbb{R}, \boldsymbol{s} \in \mathbb{R}^{2} .
$$

With this amendment, the solution of (3.40) can be written as

$$
\boldsymbol{\psi}^{\prime}\left(\left(s_{0}, \boldsymbol{s}\right),\left(t_{0}, \boldsymbol{t}\right)\right)=\left(s_{0}+t_{0}, \boldsymbol{t}+e^{-\sigma^{\top} t_{0}} \boldsymbol{s}\right) .
$$

Define the smooth invertible mapping $\phi: \mathbb{R}^{3} \rightarrow \mathbb{R}^{3}$ by

$$
\boldsymbol{\phi}\left(s_{0}, \boldsymbol{s}\right)=\left(s_{0}, e^{\sigma^{\top} s_{0}} \boldsymbol{s}\right), \quad \text { so } \boldsymbol{\phi}^{-1}\left(\boldsymbol{x}_{0}, \boldsymbol{x}\right)=\left(x_{0}, e^{-\sigma^{\top} x_{0}} \boldsymbol{x}\right),
$$

and construct an isomorphic composition function $\psi$ by using (3.27) in the form

$$
\boldsymbol{\psi}\left(\left(x_{0}, \boldsymbol{x}\right),\left(y_{0}, \boldsymbol{y}\right)\right)=\boldsymbol{\phi}\left(\boldsymbol{\psi}^{\prime}\left(\boldsymbol{\phi}^{-1}\left(x_{0}, \boldsymbol{x}\right), \boldsymbol{\phi}^{-1}\left(y_{0}, \boldsymbol{y}\right)\right)\right)
$$

This gives

$$
\boldsymbol{\psi}\left(\left(x_{0}, \boldsymbol{x}\right),\left(y_{0}, \boldsymbol{y}\right)\right)=\left(x_{0}+y_{0}, \boldsymbol{x}+e^{\sigma^{\top} x_{0}} \boldsymbol{y}\right),
$$

and we shall focus on this group composition function exclusively below. Note that if we represent the group element $\left(x_{0}, \boldsymbol{x}\right)$ as

$$
\left(\begin{array}{cccc}
e^{\sigma^{\top}} x_{0} & 0 & x_{1} \\
0 & 0 & 1 & x_{0} \\
0 & 0 & 0 & 1
\end{array}\right)
$$


then the right side of (3.45) can be written as

$$
\left(\begin{array}{cccc}
e^{\sigma^{\top}} x_{0} & 0 & x_{1} \\
0 & 0 & 0 & x_{2} \\
0 & 0 & 0 & 1
\end{array}\right)\left(\begin{array}{cccc}
e^{\sigma^{\top} y_{0}} & 0 & y_{1} \\
0 & 0 & 1 & y_{0} \\
0 & 0 & 0 & 1
\end{array}\right),
$$

so (3.46) provides a matrix representation of the group.

Note that the group composition (3.45) can also be represented in the form

$$
(g, \boldsymbol{g}) \cdot(h, \boldsymbol{h}) \equiv\left(g h, \boldsymbol{g} \phi_{g}(\boldsymbol{h})\right), \quad g, h \in \mathbb{R}, \boldsymbol{g}, \boldsymbol{h} \in \mathbb{R}^{2},
$$

where juxtaposition of elements in $\mathbb{R}$ means addition, similarly for $\mathbb{R}^{2}$, and $\phi_{g}: \mathbb{R}^{2} \rightarrow \mathbb{R}^{2}$ defined by

$$
\phi_{g}(\boldsymbol{h})=e^{\sigma^{\top} g} \boldsymbol{h},
$$

derives from (inner) automorphisms of the form

$$
(g, \mathbf{0})(0, \boldsymbol{h})(g, \mathbf{0})^{-1}=\left(0, e^{\sigma^{\top} g} \boldsymbol{h}\right) .
$$

So $\mathbb{R}$ acts on $\mathbb{R}^{2}$ via $\phi_{g}$, and (3.45) is a semi-direct product.

Now we recall some results from [13].

- If $G$ is a simply connected, connected, non-compact, non-nilpotent three-dimensional Lie group with maximal nilpotent subgroup $N$ and discrete subgroup $D$ such that $G / D$ is compact, then $\operatorname{dim} N=2$.

Note that if $G$ is defined by (3.45), then $\operatorname{dim} N=2\left(\left(\mathbb{R}^{2},+\right)\right.$ is the maximal nilpotent subgroup). Suppose then that $G$ defined by (3.45) has discrete subgroups $D$ with $G / D$ compact. We have from [13]:

- The matrix group $\left\{e^{\sigma^{\top} g}:(g, \boldsymbol{g}) \in D\right\}$ is generated by a single element $e^{\sigma^{\top} \theta},(\theta, \boldsymbol{\theta}) \in D$, $\theta \neq 0 . D \cap\left\{\left(0, \mathbb{R}^{2}\right)\right\}$ is a lattice generated by two independent elements $\left(0, \boldsymbol{e}_{1}\right),\left(0, \boldsymbol{e}_{2}\right)$, $\boldsymbol{e}_{1}, \boldsymbol{e}_{2} \in \mathbb{R}^{2}$ say. Moreover the lattice is preserved by $\phi_{\theta}: \mathbb{R}^{2} \rightarrow \mathbb{R}^{2}$, so that

$$
\phi_{\theta}\left(\boldsymbol{e}_{\alpha}\right)=\gamma_{\beta \alpha} \boldsymbol{e}_{\beta}, \quad \alpha, \beta=1,2, \gamma \equiv\left(\gamma_{\beta \alpha}\right) \in \mathrm{GL}_{2}(\mathbb{Z})
$$

Also $\left\{e^{\sigma^{\top} t}: t \in \mathbb{R}\right\}$ must be a one parameter subgroup of the unimodular group, so

$$
\operatorname{det}\left(e^{\sigma^{\top} t}\right)=e^{\left(\operatorname{tr} \sigma^{\top}\right) t}=1
$$

which implies that

$$
\operatorname{tr} \sigma=0, \quad \gamma \in \mathrm{SL}_{2}(\mathbb{Z}) .
$$

So $D$ is generated by three distinct elements $\left(0, \boldsymbol{e}_{1}\right),\left(0, \boldsymbol{e}_{2}\right),(\theta, \boldsymbol{\theta})$. Note that the inner automorphism $\xi_{\boldsymbol{h}}: G \rightarrow G$ defined by

$$
(0, \boldsymbol{h})\left(\theta^{\prime}, \boldsymbol{\theta}^{\prime}\right)(0, \boldsymbol{h})^{-1}=\left(\theta^{\prime}, \boldsymbol{\theta}^{\prime}+\left(\mathbb{1}-e^{\sigma^{\top} \theta^{\prime}}\right) \boldsymbol{h}\right)
$$

preserves the lattice generated by $\left(0, \boldsymbol{e}_{1}\right),\left(0, \boldsymbol{e}_{2}\right)$, as

$$
\xi_{\boldsymbol{h}}\left(0, \boldsymbol{\theta}^{\prime}\right)=\left(0, \boldsymbol{\theta}^{\prime}\right),
$$


and can be chosen such that

$$
\xi_{\boldsymbol{h}}(\theta, \boldsymbol{\theta})=(\theta, \boldsymbol{0})
$$

provided

$$
\operatorname{det}\left(e^{\sigma^{\top} \theta}-\mathbb{1}\right) \neq 0 .
$$

Hence, if (3.57) holds, we can assume that $D$ is generated by elements of the form $\left(0, \boldsymbol{e}_{1}\right),\left(0, \boldsymbol{e}_{2}\right),(\theta, 0)$. Also we can assume that $\theta>0$, as $(\theta, 0)^{-1}=(-\theta, 0)$.

\section{Note 1}

From (3.49) and (3.51), $e^{\sigma^{\top} \theta}$ and $\gamma$ are similar matrices, hence from (3.57), $\operatorname{det}(\gamma-\mathbb{1}) \neq 0$. If we put

$$
\gamma=\left(\begin{array}{ll}
a & b \\
c & d
\end{array}\right), \quad a, b, c, d \in \mathbb{Z},
$$

this last inequality implies

$$
a+d \neq 2 .
$$

Note 2

In the case that $a+d=\operatorname{tr} \gamma=\operatorname{tr} e^{\sigma^{\top} \theta}=2$, and (3.53) holds, from Nicks and Parry [18] one has

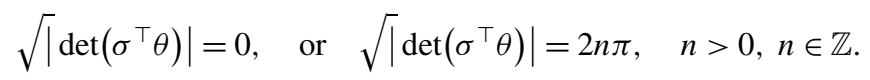

If $\operatorname{det} \sigma=0$, then since $\operatorname{tr} \sigma=0$ we have $\sigma^{2}=0$. We are considering solvable groups where $\sigma$ is similar modulo overall scaling to either $\left(\begin{array}{cc}1 & 0 \\ 0 & -1\end{array}\right),\left(\begin{array}{cc}0 & 1 \\ -1 & 0\end{array}\right)$, so $\sigma^{2} \neq 0$. Hence, when $a+d=2$, for cases of interest,

$$
\sqrt{\left|\operatorname{det}\left(\sigma^{\top} \theta\right)\right|=2 n \pi, \quad n>0, n \in \mathbb{Z}, \quad \text { and } \quad e^{\sigma^{\top} \theta}=\mathbb{1} .}
$$

\section{Note 3}

From (3.49) and (3.51)

$$
e^{\sigma^{\top} \theta} P=P \gamma, \quad P \in \mathrm{GL}_{2}(\mathbb{R}),
$$

where the columns of $P$ are $\boldsymbol{e}_{1}$ and $\boldsymbol{e}_{2}$. Hence

$$
\gamma=P^{-1} e^{\sigma^{\top} \theta} P=e^{\left(P^{-1} \sigma^{\top} P\right) \theta} \in \mathrm{SL}_{2}(\mathbb{Z})
$$

\section{Definition}

$$
M_{\sigma^{\top}}=\left\{M \in \mathrm{GL}_{2}(\mathbb{R}): M=\left(P^{-1} \sigma^{\top} P\right) \theta, P \in \mathrm{GL}_{2}(\mathbb{R}), \theta \in \mathbb{R}, \theta \neq 0\right\} .
$$

Thus $M_{\sigma^{\top}}$ consists of all matrices similar modulo overall scaling to $\sigma^{\top}$.

These remarks lead to the following proposition.

Proposition 1 Suppose $\operatorname{tr} \sigma=0$ and that there exists $\tilde{\sigma}^{\top} \in M_{\sigma^{\top}}$ such that $e^{\tilde{\sigma}^{\top}} \in S L_{2}(\mathbb{Z})$. Then $\tilde{\sigma}^{\top}=\left(P^{-1} \sigma^{\top} P\right) \theta, P \in G L_{2}(\mathbb{R}), \theta \neq 0$ and: 
(i) if $\operatorname{tr} e^{\sigma^{\top} \theta}=\operatorname{tr} e^{\tilde{\sigma}^{\top}} \neq 2,\left(0, \boldsymbol{e}_{1}\right),\left(0, \boldsymbol{e}_{2}\right),(\theta, 0)$ generate a discrete subgroup of $G$, where $\boldsymbol{e}_{1}, \boldsymbol{e}_{2}$ are the columns of $P$;

(ii) if $\operatorname{tr} e^{\sigma^{\top} \theta}=\operatorname{tr} \tilde{\sigma}^{\top}=2$, then $e^{\sigma^{\top} \theta}=\mathbb{1}$ (cf. Note 2), $e^{\tilde{\sigma}^{\top}}=\mathbb{1}$ for arbitrary $P$, and $\left(0, \boldsymbol{e}_{1}\right),\left(0, \boldsymbol{e}_{2}\right),(\theta, \boldsymbol{\theta})$ generate a discrete subgroup of $G$ whose elements coincide with the points of an arithmetic lattice with basis $\left(0, \boldsymbol{e}_{1}\right),\left(0, \boldsymbol{e}_{2}\right),(\theta, \boldsymbol{\theta}) . \boldsymbol{e}_{1}, \boldsymbol{e}_{2}$ and $\boldsymbol{\theta}$ are arbitrary, in this case (except that $\boldsymbol{e}_{1}$ and $\boldsymbol{e}_{2}$ are linearly independent).

All discrete subgroups with $G / D$ compact arise in this way. (Note that it is a short calculation, not given, to check that $\boldsymbol{\theta}$ is arbitrary in (ii).)

\subsection{Catalogue of Discrete Subgroups}

We shall list the discrete subgroups (with $G / D$ compact) according to the values of $a+d=$ $\operatorname{tr}\left(e^{\sigma^{\top} \theta}\right)$. Note first that if $A$ is any $2 \times 2$ matrix with $\operatorname{tr} A=0$, then (see Rossmann [19], for example)

$$
\begin{aligned}
& A^{2}=-\operatorname{det}(A) \mathbb{1} ; \\
& e^{A}= \begin{cases}(\cosh k) \mathbb{1}+\left(\frac{\sinh k}{k}\right) A, & \text { if } \operatorname{det} A<0, k=\sqrt{|\operatorname{det} A|}, \\
(\cos k) \mathbb{1}+\left(\frac{\sin k}{k}\right) A, & \text { if } \operatorname{det} A>0, k=\sqrt{\operatorname{det} A}, \\
\mathbb{1}+A, & \text { if } \operatorname{det} A=0 ;\end{cases} \\
& \operatorname{tr}\left(e^{A}\right)= \begin{cases}2 \cosh k, & \text { if } \operatorname{det} A<0, \\
2 \cos k, & \text { if } \operatorname{det} A>0, \\
2, & \text { if } \operatorname{det} A=0 .\end{cases}
\end{aligned}
$$

From (3.67), $a+d \geq-2$ if $e^{\sigma^{\top} \theta}$ is to lie on a one parameter subgroup of $\mathrm{SL}_{2}(\mathbb{R})$, so we identify the following cases.

(i) If $a+d>2$, from (3.67), $\operatorname{det}\left(\sigma^{\top} \theta\right)<0$. Let the eigenvalues of the traceless matrix $\sigma^{\top} \theta$ be $\lambda,-\lambda(\lambda>0)$. Then $-\lambda^{2}=\operatorname{det}\left(\sigma^{\top} \theta\right)$ so $\lambda=\sqrt{-\operatorname{det}\left(\sigma^{\top} \theta\right)}$. The eigenvalues of $e^{\sigma^{\top} \theta}$ are thus $e^{\lambda}, e^{-\lambda}$ (and are real and distinct).

(ii) If $a+d=2$, then by Note 2 above, (3.66) and (3.67), $\operatorname{det}\left(\sigma^{\top} \theta\right)>0, \sqrt{\operatorname{det}\left(\sigma^{\top} \theta\right)}=$ $2 n \pi, n \neq 0, n \in \mathbb{Z}$ and $e^{\sigma^{\top} \theta}=\mathbb{1}$ (Case $\left.C_{0}\right)$.

(iii) If $a+d \in\{-2,-1,0,+1\}$, then $\operatorname{det}\left(\sigma^{\top} \theta\right)>0$ and one calculates that $k=\sqrt{\operatorname{det}\left(\sigma^{\top} \theta\right)}$ is given by

$$
k=\left\{\begin{array}{lll}
\pi n & n= \pm 1 \bmod 2 & \text { when } a+d=-2, \\
\frac{2 \pi n}{3} & n= \pm 1 \bmod 3 & \text { when } a+d=-1, \\
\frac{\pi n}{2} & n= \pm 1 \bmod 4 & \text { when } a+d=0, \\
\frac{\pi n}{3} & n= \pm 1 \bmod 6 & \text { when } a+d=1,
\end{array}\right.
$$

and $e^{\sigma^{\top} \theta}$ may be calculated from $(3.66)_{2}$, given these values of $k$. 
Let $C_{2}, C_{3}, C_{4}, C_{6}$ be cyclic subgroups of $\mathrm{SL}_{2}(\mathbb{Z})$ generated by $-\mathbb{1},\left(\begin{array}{cc}-1 & 1 \\ -1 & 0\end{array}\right),\left(\begin{array}{cc}0 & 1 \\ -1 & 0\end{array}\right)$, $\left(\begin{array}{cc}1 & 1 \\ -1 & 0\end{array}\right)$ respectively. Then:

$$
\begin{aligned}
& \text { if } a+d=-2, \quad e^{\sigma^{\top} \theta} \text { is similar to } \gamma=-\mathbb{1}, \quad \gamma^{2}=\mathbb{1} \quad\left(\text { Case } C_{2}\right), \\
& \text { if } \left.a+d=-1, \quad e^{\sigma^{\top} \theta} \text { is similar to } \gamma=\left(\begin{array}{ll}
-1 & 1 \\
-1 & 0
\end{array}\right), \quad \gamma^{3}=\mathbb{1} \quad \text { (Case } C_{3}\right) \text {, } \\
& \text { if } a+d=0, \quad e^{\sigma^{\top} \theta} \text { is similar to } \gamma=\left(\begin{array}{cc}
0 & 1 \\
-1 & 0
\end{array}\right), \quad \gamma^{4}=\mathbb{1} \quad\left(\text { Case } C_{4}\right) \text {, } \\
& \text { if } \left.a+d=+1, \quad e^{\sigma^{\top} \theta} \text { is similar to } \gamma=\left(\begin{array}{cc}
1 & 1 \\
-1 & 0
\end{array}\right), \quad \gamma^{6}=\mathbb{1} \quad \text { (Case } C_{6}\right) \text {. }
\end{aligned}
$$

The eigenvalues of $e^{\sigma^{\top} \theta}$ are real and equal, or complex conjugate pairs, in cases (ii) and (iii) respectively, and the eigenvalues of $\sigma^{\top} \theta$ are $\pm i k$, where $k \equiv \sqrt{\operatorname{det}\left(\sigma^{\top} \theta\right)}$ takes the values given above, in case (iii).

Now recall that the matrices $\sigma$ which define the composition function (2) fall into two isomorphism classes, one class includes $\sigma=\left(\begin{array}{cc}1 & 0 \\ 0 & -1\end{array}\right)$ and all matrices in that class have real, distinct eigenvalues, the other class includes $\sigma=\left(\begin{array}{cc}0 & 1 \\ -1 & 0\end{array}\right)$ and all matrices in that class have complex conjugate eigenvalues. We shall choose particular matrices in each class (not those above, in fact), so define (two) particular composition functions, via (3.45), and construct the discrete subgroups in each case.

First we consider the group $G$ with composition function defined by $\sigma_{0}=\left(\begin{array}{cc}k_{0} & 0 \\ 0 & -k_{0}\end{array}\right)$, where $k_{0}$ is such that $e^{k_{0}}+e^{-k_{0}}=3$ (so that $e^{\sigma_{0}^{\top}}$ is similar to a matrix $\gamma_{0} \in \mathrm{SL}_{2}(\mathbb{Z})$ with $\left.\operatorname{tr} \gamma_{0}=3\right)$. We show how to construct discrete subgroups for any $\gamma \in \mathrm{SL}_{2}(\mathbb{Z})$ with $\operatorname{tr} \gamma \geq 3$. Any such $\gamma$ has unequal real eigenvalues $e^{k}, e^{-k}$ for some $k>0$, and there exists $P \in \mathrm{GL}_{2}(\mathbb{R})$ such that $\gamma=P^{-1}\left(\begin{array}{cc}e^{k} & 0 \\ 0 & e^{-k}\end{array}\right) P$. Define $\tilde{\sigma}^{\top}=P^{-1}\left(\begin{array}{cc}k & 0 \\ 0 & -k\end{array}\right) P$, so that $e^{\tilde{\sigma}^{\top}}=\gamma$. Then $\tilde{\sigma}^{\top}$ is similar modulo overall scaling to $\sigma_{0}^{\top}$, for

$$
\tilde{\sigma}^{\top}=P^{-1}\left(\begin{array}{cc}
k & 0 \\
0 & -k
\end{array}\right) P=\left(P^{-1}\left(\begin{array}{cc}
k_{0} & 0 \\
0 & -k_{0}
\end{array}\right) P\right)\left(\frac{k}{k_{0}}\right)=\left(P^{-1} \tilde{\sigma}_{0}^{\top} P\right)\left(\frac{k}{k_{0}}\right) .
$$

Then by (i) of Proposition 1, $G$ has a discrete subgroup $D$ (with $G / D$ compact) generated by $\left(0, \boldsymbol{e}_{1}\right),\left(0, \boldsymbol{e}_{2}\right),\left(\frac{k}{k_{0}}, 0\right)$, where $\boldsymbol{e}_{1}, \boldsymbol{e}_{2}$ are the columns of $P$.

Second we describe the discrete subgroups of that $G$ with composition function defined by $\sigma_{0}^{\top}=\left(\begin{array}{cc}0 & 2 \pi \\ -2 \pi & 0\end{array}\right)$, so that $e^{\sigma_{0}^{\top}}=\mathbb{1}$. We show how to construct discrete subgroups for any $\gamma \in \mathrm{SL}_{2}(\mathbb{Z})$ with $-2 \leq \operatorname{tr} \gamma \leq 2$. Any such matrix is similar in $\mathrm{GL}_{2}(\mathbb{R})$ to one of the unimodular integer matrices listed in cases (ii), (iii) above (cases $C_{0}, C_{2}, C_{3}$, $C_{4}, C_{6}$ ), and likewise similar to $e^{\sigma^{\dagger \top}}$, where $\sigma^{\dagger \top}=\left(\begin{array}{cc}0 & k \\ -k & 0\end{array}\right)$ with $k=2 n \pi$ ( $n$ a positive integer) in case (ii), $k$ given by (3.68) in case (iii). Thus there exists $P \in \mathrm{GL}_{2}(\mathbb{R})$ such that $\gamma=P^{-1} e^{\sigma^{\dagger \top}} P=e^{P^{-1} \sigma^{\dagger \top} P} \in \mathrm{SL}_{2}(\mathbb{Z})$. So $\gamma=e^{\tilde{\sigma}^{\top}}$ where

$$
\tilde{\sigma}^{\top}=P^{-1} \sigma^{\dagger \top} P=P^{-1}\left(\begin{array}{cc}
0 & k \\
-k & 0
\end{array}\right) P=P^{-1}\left(\begin{array}{cc}
0 & 2 \pi \\
-2 \pi & 0
\end{array}\right) P\left(\frac{k}{2 \pi}\right)=P^{-1} \sigma_{0}^{\top} P\left(\frac{k}{2 \pi}\right) .
$$

So, in the case that $a+d=2, G$ has discrete subgroups generated by $\left(0, \boldsymbol{e}_{1}\right),\left(0, \boldsymbol{e}_{2}\right),(n, \boldsymbol{\theta})$, where $\boldsymbol{e}_{1}, \boldsymbol{e}_{2}, \boldsymbol{\theta}$ are arbitrary, $n>0, n \in \mathbb{Z}\left(e^{\sigma^{\dagger \top}}=\mathbb{1}\right.$ in this case, so $P$ is arbitrary). When 
$-2 \leq a+d<2, G$ has discrete subgroups generated by $\left(0, \boldsymbol{e}_{1}\right),\left(0, \boldsymbol{e}_{2}\right),\left(\frac{k}{2 \pi}, 0\right)$, where $\boldsymbol{e}_{1}, \boldsymbol{e}_{2}$ are the columns of $P$, and from (3.68), $\frac{k}{2 \pi}= \pm \frac{1}{p} \bmod \mathbb{Z}$ in the cases denoted $C_{p}$, $p=2,3,4,6$ in (iii) above.

\section{Canonical Forms of Vector Fields in $\mathbb{R}^{2}$ with Three-Dimensional Solvable Lattice Algebras}

Here we construct canonical forms of the vector fields in two ways, first using homogeneous space methods, second using direct analytical methods-we give the two methods in order to convince the reader that the first method is the more systematic and informative of the two. We restrict to vector fields where the corresponding solvable Lie algebra is unimodular, so we do not discuss the case $\operatorname{dim} \boldsymbol{g}_{2}=1$, nor the case where $\sigma$ is non-diagonalizable and $\operatorname{dim} \boldsymbol{g}_{2}=2$. Thus we take $\sigma=\left(\begin{array}{cc}1 & 0 \\ 0 & -1\end{array}\right),\left(\begin{array}{cc}0 & 1 \\ -1 & 0\end{array}\right)$.

\subsection{Homogeneous Space Construction}

(i) Recall from the remarks following (3.31) that if $\sigma=\left(\begin{array}{cc}1 & 0 \\ 0 & -1\end{array}\right)$ there are three onedimensional subalgebras of $\mathfrak{g}$, with basis $(1,0,0)^{\top},(0,1,0)^{\top},(0,1,1)^{\top}$ respectively, which are inequivalent modulo algebra automorphism. From (2.28), the corresponding group automorphism produces the inequivalent one-dimensional subgroups $e^{\left\langle(1,0,0)^{\top}\right\rangle}$, $e^{\left\langle(0,1,0)^{\top}\right\rangle}, e^{\left\langle(0,1,1)^{\top}\right\rangle}$. One can calculate the corresponding group actions using Sect. 2.6. Finally one finds the (three sets of) infinitesimal generators, which are the canonical forms of the vector fields, when $\sigma=\left(\begin{array}{cc}1 & 0 \\ 0 & -1\end{array}\right)$. Let $\boldsymbol{e}_{0} \equiv(1,0,0)^{\top}$, etc., in this section.

- The elements of the one parameter subgroup $H=\{\boldsymbol{x}(t) ; t \in \mathbb{R}\}$ corresponding to $\left\langle\boldsymbol{e}_{0}\right\rangle$ coincide with the points of the integral curve of the right invariant field whose components at the group identity are $\boldsymbol{e}_{0} \equiv(1,0,0)^{\top}$. So

$$
\frac{d}{d t} \boldsymbol{x}(t)=\nabla_{1} \psi(0, x(t)) \boldsymbol{e}_{0},
$$

where $\boldsymbol{\psi}$ is given by (3.45). This gives $H=\left\{t \boldsymbol{e}_{0} ; t \in \mathbb{R}\right\}$. If $\boldsymbol{g}=(z, x, y) \in G$, the left cosets have the form

$$
\boldsymbol{g} H=\{\boldsymbol{\psi}((z, x, y),(t, 0,0)) ; t \in \mathbb{R}\}=\{(z+t, x, y): t \in \mathbb{R}\} .
$$

So elements of the set of left cosets may be identified by the two coordinates $(x, y) \in \mathbb{R}^{2}$. Define the projection $\pi: G \rightarrow G / H \equiv \mathbb{R}^{2}$ by

$$
\boldsymbol{\pi}(z, x, y)=(x, y)
$$

and choose a corresponding section $\sigma: G / H \equiv \mathbb{R}^{2} \rightarrow G$ by

$$
\boldsymbol{\sigma}(x, y)=(0, x, y) \text {. }
$$

(For consistency with (3.41), we should write these last two equations as $\pi(z, x, y)=$ $\left(\begin{array}{l}x \\ y\end{array}\right), \sigma\left(\begin{array}{l}x \\ y\end{array}\right)=(0, x, y)$, but is convenient to allow a little notational abuse in this subsection.) 
Then according to (2.45), with $\boldsymbol{g}_{1} \equiv\left(z_{1}, x_{1}, y_{1}\right)$, the corresponding group action $\lambda: G \times \mathbb{R}^{2} \rightarrow \mathbb{R}^{2}$ is

$$
\begin{aligned}
\lambda\left(\boldsymbol{g}_{1}, \boldsymbol{g} H\right)=\boldsymbol{\pi}\left(\boldsymbol{g}_{1} \boldsymbol{\sigma}(\boldsymbol{g} H)\right) & =\boldsymbol{\pi}\left(\boldsymbol{\psi}\left(\left(z_{1}, x_{1}, y_{1}\right),(0, x, y)\right)\right) \\
& =\pi\left(\left(z_{1}, x_{1}+\alpha, y_{1}+\beta\right)\right) \\
& =\left(x_{1}+\alpha, y_{1}+\beta\right),
\end{aligned}
$$

where

$$
\left(\begin{array}{l}
\alpha \\
\beta
\end{array}\right)=e^{\sigma^{\top} z_{1}}\left(\begin{array}{l}
x \\
y
\end{array}\right), \quad \sigma=\left(\begin{array}{cc}
1 & 0 \\
0 & -1
\end{array}\right) .
$$

The three infinitesimal generators $l_{0}(\cdot), l_{1}(\cdot), l_{2}(\cdot)$ (corresponding to the choices $\left.\boldsymbol{v}=\boldsymbol{e}_{i}, i=0,1,2\right)$ have components given by

$$
\frac{\partial \lambda_{r}}{\partial\left(\boldsymbol{g}_{1}\right)_{i}}(\mathbf{0},(x, y)) \equiv \frac{\partial \lambda_{r}}{\partial\left(\boldsymbol{g}_{1}\right)_{i}}(\mathbf{0}, \boldsymbol{g} H), \quad r=1,2, i=0,1,2,
$$

where $\left(\lambda_{1}, \lambda_{2}\right)$ are the components of $\lambda$. It follows that

$$
\boldsymbol{l}_{0}(x, y)=(x,-y), \quad \boldsymbol{l}_{1}(x, y)=(1,0), \quad \boldsymbol{l}_{2}(x, y)=(0,1) .
$$

- For the subalgebra $\left\langle\boldsymbol{e}_{1}+\boldsymbol{e}_{2}\right\rangle$, one obtains $H=\{(0, r, r): r \in \mathbb{R}\}$, and so with $\boldsymbol{g}=$ $\left(t_{0}, t_{1}, t_{2}\right)$,

$$
\boldsymbol{g} H=\left\{\left(t_{0}, \tau_{1}, \tau_{2}\right):\left(\begin{array}{l}
\tau_{1} \\
\tau_{2}
\end{array}\right)=\left(\begin{array}{l}
t_{1} \\
t_{2}
\end{array}\right)+r\left(\begin{array}{c}
e^{t_{0}} \\
e^{-t_{0}}
\end{array}\right), r \in \mathbb{R}\right\} .
$$

For fixed $t_{0}, t_{1}, t_{2}$, (4.8) represents a line through $\left(\begin{array}{l}t_{1} \\ t_{2}\end{array}\right)$ also passing through the point $\frac{t_{1} e^{-t_{0}}-t_{2} e^{t_{0}}}{e^{2 t_{0}}+e^{-2 t_{0}}}\left(\begin{array}{c}e^{-t_{0}} \\ -e^{t_{0}}\end{array}\right)$. Note that this line has signed distance $\left(t_{1} e^{-t_{0}}-\right.$ $\left.t_{2} e^{t_{0}}\right) / \sqrt{e^{2 t_{0}}+e^{-2 t_{0}}}$ from the origin in the $t_{0}$ plane. So we can identify $g H$ as a point of $\mathbb{R}^{2}$ with coordinates

$$
\boldsymbol{g} H \equiv\left(t_{0}, t_{1} e^{-t_{0}}-t_{2} e^{t_{0}}\right),
$$

and choose as projection mapping $\pi: G \rightarrow G / H \equiv \mathbb{R}^{2}$,

$$
\boldsymbol{\pi}\left(t_{0}, t_{1}, t_{2}\right)=\left(t_{0}, t_{1} e^{-t_{0}}-t_{2} e^{t_{0}}\right)
$$

As choice of section $\sigma: G / H \equiv \mathbb{R}^{2} \rightarrow G$ we take

$$
\sigma(x, y)=\left(x, \frac{y e^{-x}}{e^{2 x}+e^{-2 x}}, \frac{-y e^{x}}{e^{2 x}+e^{-2 x}}\right),
$$

as $\sigma$ maps $\pi\left(t_{0}, t_{1}, t_{2}\right)$ to the point identified after (4.8), which is the closest point, on the line represented by (4.8), to the origin in the plane $t_{0}=$ constant. We calculate the 
group action, with $\boldsymbol{g}_{1}=\left(z_{0}, z_{1}, z_{2}\right)$,

$$
\begin{aligned}
\lambda\left(\boldsymbol{g}_{1} \boldsymbol{g} H\right) & =\boldsymbol{\pi}\left(\boldsymbol{g}_{1} \boldsymbol{\sigma}(\boldsymbol{g} H)\right)=\boldsymbol{\pi}\left(\boldsymbol{\psi}\left(\left(z_{0}, z_{1}, z_{2}\right),\left(x, \frac{y e^{-x}}{e^{2 x}+e^{-2 x}}, \frac{-y e^{x}}{e^{2 x}+e^{-2 x}}\right)\right)\right) \\
& =\pi\left(z_{0}+x, z_{1}+\tau_{1}, z_{2}+\tau_{2}\right) \\
& =\left(z_{0}+x,\left(z_{1}+\tau_{1}\right) e^{-\left(z_{0}+x\right)}-\left(z_{2}+\tau_{2}\right) e^{z_{0}+x}\right) \\
& =\left(z_{0}+x, y+z_{1} e^{-z_{0}-x}-z_{2} e^{z_{0}+x}\right),
\end{aligned}
$$

where we used

$$
\left(\begin{array}{l}
\tau_{1} \\
\tau_{2}
\end{array}\right)=\left(\begin{array}{cc}
e^{z_{0}} & 0 \\
0 & e^{-z_{0}}
\end{array}\right)\left(\begin{array}{c}
y e^{-x} \\
-y e^{x}
\end{array}\right) /\left(e^{2 x}+e^{-2 x}\right) .
$$

One finds that the infinitesimal generators are:

$$
\boldsymbol{l}_{0}=(1,0), \quad \boldsymbol{l}_{1}=\left(0, e^{-x}\right), \quad \boldsymbol{l}_{2}=\left(0,-e^{x}\right) .
$$

- For the subalgebra $\left\langle\boldsymbol{e}_{1}\right\rangle, H=\{(0, r, 0): r \in \mathbb{R}\}$, and if $\boldsymbol{g}=\left(t_{0}, t_{1}, t_{2}\right)$ then $\boldsymbol{g} H=$ $\left\{\left(t_{0}, t_{1}+r e^{t_{0}}, t_{2}\right): r \in \mathbb{R}\right\}$. For fixed $t_{0}$, the points of $\boldsymbol{g} H$ represent a line through $\left(\begin{array}{c}t_{1} \\ t_{2}\end{array}\right)$ also passing through $\left(\begin{array}{c}0 \\ t_{2}\end{array}\right)$. So we identify $\boldsymbol{g} H \equiv\left(t_{0}, t_{2}\right)$, set

$$
\boldsymbol{\pi}\left(t_{0}, t_{1}, t_{2}\right)=\left(t_{0}, t_{2}\right),
$$

and choose

$$
\boldsymbol{\sigma}(x, y)=(x, 0, y) .
$$

We calculate, with $\boldsymbol{g}_{1}=\left(z_{0}, z_{1}, z_{2}\right)$;

$$
\begin{aligned}
\lambda\left(\boldsymbol{g}_{1} \boldsymbol{g} H\right) & =\boldsymbol{\pi}\left(\boldsymbol{g}_{1} \boldsymbol{\sigma}(\boldsymbol{g} H)\right)=\boldsymbol{\pi}\left(\boldsymbol{\psi}\left(\left(z_{0}, z_{1}, z_{2}\right),(x, 0, y)\right)\right) \\
& =\boldsymbol{\pi}\left(z_{0}+x, z_{1}+\tau_{1}, z_{2}+\tau_{2}\right), \quad \text { where }\left(\begin{array}{c}
\tau_{1} \\
\tau_{2}
\end{array}\right)=\left(\begin{array}{cc}
e^{z_{0}} & 0 \\
0 & e^{-z_{0}}
\end{array}\right)\left(\begin{array}{l}
0 \\
y
\end{array}\right) \\
& =\pi\left(z_{0}+x, z_{1}, z_{2}+y e^{-z_{0}}\right) \\
& =\left(z_{0}+x, z_{2}+y e^{-z_{0}}\right) .
\end{aligned}
$$

Finally, the infinitesimal generators are:

$$
l_{0}(x, y)=(1,-y), \quad l_{1}(x, y)=(0,0), \quad l_{3}(x, y)=(0,1) .
$$

Since this is a 2-dimensional algebra of vector fields, we consider it no further.

(ii) - If $\sigma=\left(\begin{array}{cc}0 & 1 \\ -1 & 0\end{array}\right)$, and (recalling the remark after (3.32)) we consider the subalgebra $\left\langle\boldsymbol{e}_{0}\right\rangle$, then by the analogue of (4.1) we get $H=\left\{t \boldsymbol{e}_{0}: t \in \mathbb{R}\right\}$. Then $\boldsymbol{g} H=$ $\{(z+t, x, y): t \in \mathbb{R}\}$ if $\boldsymbol{g}=(z, x, y)$ and we can take $\boldsymbol{\pi}(z, x, y)=(x, y), \boldsymbol{\sigma}(x, y)=$ $(0, x, y)$. With $g_{1}=\left(z_{1}, x_{1}, y_{1}\right)$ one finds that

$$
\lambda\left(\boldsymbol{g}_{1}, \boldsymbol{g} H\right)=\left(x_{1}+x \cos z_{1}-y \sin z_{1}, y_{1}+x \sin z_{1}+y \cos z_{1}\right),
$$


and calculates that the infinitesimal generators are:

$$
l_{0}(x, y)=(-y, x), \quad l_{1}(x, y)=(1,0), \quad l_{2}(x, y)=(0,1) .
$$

- For the subalgebra $\left\langle\boldsymbol{e}_{1}\right\rangle$, we get $H=\left\{t \boldsymbol{e}_{1}: t \in \mathbb{R}\right\}$, and with $\boldsymbol{g}=(z, x, y), \boldsymbol{g} H=$ $\{(z, x+t \cos z, y+t \sin z): t \in \mathbb{R}\}$. For fixed $(z, x, y), g H$ represents a line in the plane $z=$ constant, through $(z, x, y)$, making an angle $z$ with the $x$-axis. The point nearest the origin (i.e., nearest $(z, 0,0))$ is $(z,(y \cos z-x \sin z)(-\sin z, \cos z))$. Therefore we can identify $g H$ by

$$
\boldsymbol{\pi}(\boldsymbol{g})=(z, y \cos z-x \sin z),
$$

and choose as section the point nearest the origin, so

$$
\sigma(x, y)=(x,-y \sin x, y \cos x) .
$$

One calculates, in the manner of the previous bullet points, that with $g_{1}=\left(z_{1}, x_{1}, y_{1}\right)$,

$$
\lambda\left(\boldsymbol{g}_{1}, \boldsymbol{g} H\right)=\left(z_{1}+z, y \cos z-x \sin z-x_{1} \sin \left(z_{1}+z\right)+y_{1} \cos \left(z_{1}+z\right)\right) .
$$

Finally the infinitesimal generators are:

$$
\boldsymbol{l}_{0}(x, y)=(1,0), \quad \boldsymbol{l}_{1}(x, y)=(0,-\sin x), \quad \boldsymbol{l}_{2}(x, y)=(0, \cos x) .
$$

Note that the change of basis $\boldsymbol{l}_{0} \rightarrow \boldsymbol{l}_{0}, \boldsymbol{l}_{1} \rightarrow+\boldsymbol{l}_{2}, \boldsymbol{l}_{2} \rightarrow-\boldsymbol{l}_{1}$ does not change the commutation relations, so the infinitesimal generators can also be written as

$$
\boldsymbol{l}_{0}(x, y)=(1,0), \quad \boldsymbol{l}_{1}(x, y)=(0, \cos x), \quad \boldsymbol{l}_{2}(x, y)=(0, \sin x) .
$$

\subsection{Direct Construction of Canonical Vector Fields}

We construct vector fields $\boldsymbol{v}_{0}, \boldsymbol{v}_{1}, \boldsymbol{v}_{2}$ satisfying (cf. (3.18)):

$$
\left[\boldsymbol{v}_{1}, \boldsymbol{v}_{2}\right](\cdot)=\mathbf{0}, \quad\left[\boldsymbol{v}_{0}, \boldsymbol{v}_{\alpha}\right]=\sigma_{\alpha \beta} \boldsymbol{v}_{\beta}(\cdot), \quad \alpha, \beta=1,2,
$$

for $\sigma=\left(\sigma_{\alpha \beta}\right)=\left(\begin{array}{cc}1 & 0 \\ 0 & -1\end{array}\right)$ and $\left(\begin{array}{cc}0 & 1 \\ -1 & 0\end{array}\right)$. The vector fields are to coincide with the infinitesimal generators of a transitive three-dimensional group, so from [5], Cor. 2.66, the dimension of $\left\langle\boldsymbol{v}_{0}, \boldsymbol{v}_{1}, \boldsymbol{v}_{2}\right\rangle$ is two at each point. We assume that the components of the vector fields are analytic functions of $(x, y) \in \mathbb{R}^{2}$, and focus on a neighbourhood of the origin in $\mathbb{R}^{2}$. Thus we can assume that at least one of $\boldsymbol{v}_{1}(0,0), \boldsymbol{v}_{2}(0,0) \neq \mathbf{0}$. Suppose that $\boldsymbol{v}_{1}(0,0) \neq 0$. Then we can choose local coordinates so that $\boldsymbol{v}_{1}(x, y)=(1,0)^{\top}$, and since $\left[\boldsymbol{v}_{1}, \boldsymbol{v}_{2}\right](\cdot) \equiv 0$ it follows that $\boldsymbol{v}_{2}(x, y)=(\alpha(y), \beta(y))^{\top}$, for some functions $\alpha(\cdot), \beta(\cdot)$. There are two cases, depending on whether or not $\boldsymbol{v}_{1}(0,0)$ and $\boldsymbol{v}_{2}(0,0)$ are linearly dependent, i.e., depending on whether or not $\beta(0) \neq 0$.

(i) $\beta(0) \neq 0$

Define $g(y), f(y)$ by

$$
g^{\prime}(y)=\frac{1}{\beta(g)}, \quad f^{\prime}(y)=-\frac{\alpha(y)}{\beta(y)}, \quad f(0)=g(0)=0,
$$


and a change of coordinates $(x, y) \rightarrow(\tilde{x}, \tilde{y})$ by

$$
\tilde{x}=x+f(y), \quad \tilde{y}=g(y) .
$$

Then from (1.4) we get

$$
\tilde{\boldsymbol{v}}_{2}(\tilde{x}, \tilde{y})=\left(\begin{array}{ll}
1 & f^{\prime}(y) \\
0 & g^{\prime}(y)
\end{array}\right)\left(\begin{array}{c}
\alpha(y) \\
\beta(y)
\end{array}\right)=\left(\begin{array}{l}
0 \\
1
\end{array}\right), \quad \tilde{\boldsymbol{v}}_{1}(\tilde{x}, \tilde{y})=\left(\begin{array}{l}
1 \\
0
\end{array}\right) .
$$

Dropping the tilde, for convenience, we now have

$$
\boldsymbol{v}_{1}=(1,0)^{\top}, \quad \boldsymbol{v}_{2}=(0,1)^{\top}, \quad \boldsymbol{v}_{0}=(c(x, y), d(x, y))^{\top},
$$

say. So from (4.25)

$$
\left(\begin{array}{l}
\frac{\partial c}{\partial x}, \frac{\partial d}{\partial x} \\
\frac{\partial c}{\partial y}, \frac{\partial d}{\partial y}
\end{array}\right)=\sigma=\left(\sigma_{\alpha \beta}\right) .
$$

Thus $\boldsymbol{v}_{0}=\left(\sigma_{11} x+\sigma_{21} y+c_{0}, \sigma_{12} x+\sigma_{22} y+d_{0}\right)^{\top}, c_{0}, d_{0} \in \mathbb{R}$. Since adding a linear combination of $\boldsymbol{v}_{1}(\cdot)$ and $\boldsymbol{v}_{2}(\cdot)$ to $\boldsymbol{v}_{0}(\cdot)$ does not change the commutation relations (4.25), we can assume that $c_{0}=d_{0}=0$. Thus

$$
\boldsymbol{v}_{0}(x, y)=\sigma^{\top}\left(\begin{array}{l}
x \\
y
\end{array}\right)
$$

Hence we arrive at the vector fields given in (4.7) and (4.19) (bearing in mind the remark after (4.4)).

(ii) $\beta(0)=0$

In this case $\boldsymbol{v}_{0}(0,0) \neq \mathbf{0}$, so we put $\boldsymbol{v}_{0}(x, y)=(1,0)^{\top}$ and

$$
\boldsymbol{v}_{1}(x, y)=(a(x, y), b(x, y))^{\top}, \quad \boldsymbol{v}_{2}(x, y)=(c(x, y), d(x, y))^{\top} .
$$

Then from (4.25),

$$
-\frac{\partial}{\partial x}(a, b)^{\top}=\sigma_{11}(a, b)^{\top}+\sigma_{12}(c, d)^{\top}, \quad-\frac{\partial}{\partial x}(c, d)^{\top}=\sigma_{21}(a, b)^{\top}+\sigma_{22}(c, d)^{\top},
$$

and rearranging

$$
-\frac{\partial}{\partial x}\left(\begin{array}{l}
a \\
c
\end{array}\right)=\sigma\left(\begin{array}{l}
a \\
c
\end{array}\right), \quad-\frac{\partial}{\partial x}\left(\begin{array}{l}
b \\
d
\end{array}\right)=\left(\begin{array}{l}
b \\
d
\end{array}\right) .
$$

So

$$
\left(\begin{array}{ll}
a & b \\
c & d
\end{array}\right)=\exp (-\sigma x) D(y)
$$

for some matrix $D(y)$.

- In the case that $\sigma=\left(\begin{array}{cc}1 & 0 \\ 0 & -1\end{array}\right),(4.35)$ gives

$$
\boldsymbol{v}_{1}(x, y)=\left(\alpha(y) e^{-x}, \bar{\beta}(y) e^{-x}\right)^{\top}, \quad \boldsymbol{v}_{2}(x, y)=\left(\gamma(y) e^{x}, \delta(y) e^{x}\right)^{\top},
$$


for some functions $\alpha, \bar{\beta}, \gamma, \delta$ (the functions $\beta(\cdot)$ and $\bar{\beta}(\cdot)$ are unrelated). Now one of $\bar{\beta}(0), \delta(0)$ is non-zero (since $\operatorname{dim}\left\langle\boldsymbol{v}_{0}, \boldsymbol{v}_{1}, \boldsymbol{v}_{2}\right\rangle=2$ ). Suppose that $\bar{\beta}(0) \neq 0$, without loss of generality. By change of coordinates $x \rightarrow \tilde{x}=x+f(y), y \rightarrow \tilde{y}=g(y)$, where $f^{\prime}=-\alpha / \bar{\beta}, g^{\prime}=e^{-f(y)} / \bar{\beta}$, we get that $\boldsymbol{v}_{1}=\left(0, e^{-x}\right)^{\top}$ and that $\boldsymbol{v}_{2}$ has the functional form given in (4.35), in the new coordinates. Then $(4.25)_{1}$ gives

$$
\left[\left(0, e^{-x}\right)^{\top},\left(\gamma(y) e^{x}, \delta(y) e^{x}\right)^{\top}\right]=\mathbf{0},
$$

which implies that $\gamma^{\prime}(y)=0,-\gamma(y)=\delta^{\prime}(y)$. Thus $\gamma(y)=0$, since if $\gamma(0) \neq 0$, $\boldsymbol{v}_{1}$ and $\boldsymbol{v}_{2}$ are linearly independent at the origin. So $\delta(y)$ is a constant, and we may presume that $\delta(y)=-1$ (cf. (3.30)). Thus

$$
\boldsymbol{v}_{0}(x, y)=(1,0)^{\top}, \quad \boldsymbol{v}_{1}(x, y)=\left(0, e^{-x}\right)^{\top}, \quad \boldsymbol{v}_{2}(x, y)=\left(0,-e^{x}\right)^{\top},
$$

and these are the vector fields given in (4.13).

- In the case that $\sigma=\left(\begin{array}{cc}0 & 1 \\ -1 & 0\end{array}\right)$, then (4.35) gives

$$
\left(\begin{array}{ll}
a & b \\
c & d
\end{array}\right)=\left(\begin{array}{cc}
\cos x & -\sin x \\
\sin x & \cos x
\end{array}\right) D(y)=R(x)\left(\begin{array}{ll}
\alpha(y) & \beta(y) \\
\gamma(y) & \delta(y)
\end{array}\right),
$$

where $R(x), D(y)$ are defined in the obvious way (new function $\beta(\cdot)$ ). So

$$
\begin{aligned}
& \boldsymbol{v}_{1}(x, y)=(\alpha \cos x-\gamma \sin x, \beta \cos x-\delta \sin x)^{\top}, \\
& \boldsymbol{v}_{2}(x, y)=(\alpha \sin x+\gamma \cos x, \beta \sin x+\delta \cos x)^{\top} .
\end{aligned}
$$

At least one of $\beta(0), \alpha(0)$ is non-zero at the origin, without loss of generality assume that $\beta(0) \neq 0$. Let $\tilde{x}=x+f(y), \tilde{y}=g(y)$ and let $(\tilde{a}(\tilde{x}, \tilde{y}), \tilde{b}(\tilde{x}, \tilde{y}))^{\top}$, $(\tilde{c}(\tilde{x}, \tilde{y}), \tilde{d}(\tilde{x}, \tilde{y}))^{\top}$ be the components of $\boldsymbol{v}_{1}, \boldsymbol{v}_{2}$ in the new coordinates. Then

$$
\begin{aligned}
&\left(\begin{array}{cc}
\tilde{a} & \tilde{c} \\
\tilde{b} & \tilde{d}
\end{array}\right)_{\tilde{x}=\tilde{x}(x, y), \tilde{y}=\tilde{y}(y)} \\
&=\left(\begin{array}{cc}
1 & f^{\prime}(y) \\
0 & g^{\prime}(y)
\end{array}\right)\left(\begin{array}{cc}
\alpha(y) & \gamma(y) \\
\beta(y) & \delta(y)
\end{array}\right) R(-x), \\
&=\left(\begin{array}{cc}
\alpha+\beta f^{\prime} & \gamma+\delta f^{\prime} \\
\beta g^{\prime} & \delta g^{\prime}
\end{array}\right) R(f) R(-x-f) \\
&=\left(\begin{array}{cc}
\left(\alpha+\beta f^{\prime}\right) \cos f+\left(\gamma+\delta f^{\prime}\right) \sin f & -\left(\alpha+\beta f^{\prime}\right) \sin f+\left(\gamma+\delta f^{\prime}\right) \cos f \\
g^{\prime}(\beta \cos f+\delta \sin f) & g^{\prime}(-\beta \sin f+\delta \cos f)
\end{array}\right) \\
& R(-x-f) .
\end{aligned}
$$

Now choose $f$ so that the 11 component of the matrix displayed in the line above is zero, recalling $\beta(0) \neq 0$

$$
f^{\prime}=-\frac{(\alpha \cos f+\gamma \sin f)}{\beta \cos f+\delta \sin f}, \quad f(0)=0 .
$$

Knowing $f=f(y)$, choose $g$ so that the 21 component of the matrix is 1 :

$$
g^{\prime}=\frac{1}{\beta \cos f+\delta \sin f}, \quad g(0)=0 .
$$


Then from (4.40)

$$
\left(\begin{array}{ll}
\tilde{a} & \tilde{c} \\
\tilde{b} & \tilde{d}
\end{array}\right)_{\tilde{x}, \tilde{y}}=\left(\begin{array}{cc}
0 & \xi(\tilde{y}) \\
1 & \mu(\tilde{y})
\end{array}\right) R(-\tilde{x}),
$$

for some functions $\xi, \mu$, and dropping the tilde this gives

$$
\begin{aligned}
& \boldsymbol{v}_{1}(x, y)=(-\xi(y) \sin x, \cos x-\mu(y) \sin x)^{\top}, \\
& \boldsymbol{v}_{2}(x, y)=(\xi(y) \cos x, \sin x+\mu(y) \cos x)^{\top} .
\end{aligned}
$$

Since $\boldsymbol{v}_{1}$ and $\boldsymbol{v}_{2}$ are linearly dependent at the origin, $\xi(0)=0$. We calculate that $\left[\boldsymbol{v}_{1}, \boldsymbol{v}_{2}\right](\cdot)$ has components $-\left(\xi^{\prime}+\xi^{2}, \mu^{\prime}+\xi \mu\right)$. Hence $\xi^{\prime}+\xi^{2}=0, \xi(0)=0$, so $\xi(y)=0, \mu^{\prime}(y)=0$. So $\mu(y)=\mu_{0} \in \mathbb{R}$ and $\boldsymbol{v}_{1}(x, y)=\left(0, \cos x-\mu_{0} \sin x\right)^{\top}, \boldsymbol{v}_{2}=$ $\left(0, \sin x+\mu_{0} \cos x\right)^{\top}$. Now from (3.31) we may replace $\boldsymbol{v}_{1}$ by $\tilde{\boldsymbol{v}}_{1}=\alpha \boldsymbol{v}_{1}-\beta \boldsymbol{v}_{2}, \boldsymbol{v}_{2}$ by $\tilde{\boldsymbol{v}}_{2}=\beta \boldsymbol{v}_{1}+\alpha \boldsymbol{v}_{2}, \alpha, \beta \in \mathbb{R}, \alpha^{2}+\beta^{2}>0$ without changing the commutation relations.

By putting $\alpha=\left(1+\mu_{0}^{2}\right)^{-1}, \beta=-\mu_{0} \alpha$ we obtain

$$
\boldsymbol{v}_{0}(x, y)=(1,0)^{\top}, \quad \boldsymbol{v}_{1}(x, y)=(0, \cos x)^{\top}, \quad \boldsymbol{v}_{2}(x, y)=(0, \sin x)^{\top},
$$

and so recover (4.24).

\section{Sets of Points Obtained by Discrete Flow Along the Primary Lattice Vector Fields}

Given the two-dimensional crystal state $\Sigma,(1.18)$, defined by vector fields $l_{0}(\cdot), l_{1}(\cdot)$ generating a three-dimensional lattice algebra, construct a set of points $S_{\Sigma}$ in $\Omega$ by the following iterative procedure. Let $\varepsilon, \mu \in \mathbb{R}$ both be positive. Choose a point $\boldsymbol{x}_{0} \in \Omega$ as starting point and construct two points $\boldsymbol{x}(\varepsilon), \boldsymbol{x}(-\varepsilon) \in \Omega$ by solving

$$
\frac{d x}{d t}(t)=l_{0}(x(t)), \quad x(0)=x_{0}, t \in \mathbb{R}
$$

Obtain two further points $\boldsymbol{y}(\mu), \boldsymbol{y}(-\mu) \in \Omega$ by solving

$$
\frac{d \boldsymbol{y}}{d s}(s)=\boldsymbol{l}_{1}(\boldsymbol{y}(s)), \quad \boldsymbol{y}(0)=\boldsymbol{x}_{0}, s \in \mathbb{R} .
$$

Iterate this process, using the four points $\boldsymbol{x}(\varepsilon), \boldsymbol{x}(-\varepsilon), \boldsymbol{y}(\mu), \boldsymbol{y}(-\mu)$ so obtained as starting points in turn, to begin with. Continue indefinitely in this way and denote the set of all points obtained by $S_{\Sigma} . S_{\Sigma}$ depends on $\varepsilon, \mu$. We use (2.48), (2.49) to represent $S_{\Sigma}$. So put $\boldsymbol{g}=\mathbf{0}$ in those equations to deduce that $\boldsymbol{x}(t)=\lambda\left(e^{t e_{0}}, \boldsymbol{x}_{0}\right)$ solves (5.1), as the Lie algebra element corresponding to $\boldsymbol{l}_{0}(\cdot)$ is $\boldsymbol{e}_{0}$ (cf. (4.6), for example). Let $\boldsymbol{\pi}, \boldsymbol{\sigma}$ be the projection and section mappings deriving from a particular choice of isotropy group $H$, and corresponding group action, so $\lambda(g, x)=\pi(g \sigma(x))$. Then

$$
\boldsymbol{x}(\varepsilon)=\lambda\left(e^{\varepsilon \boldsymbol{e}_{0}}, \boldsymbol{x}_{0}\right)=\boldsymbol{\pi}\left(e^{\varepsilon \boldsymbol{e}_{0}} \boldsymbol{\sigma}\left(\boldsymbol{x}_{0}\right)\right)=\boldsymbol{\pi}\left(e^{\varepsilon \boldsymbol{e}_{0}} \boldsymbol{g}_{0}\right),
$$

where we define

$$
\boldsymbol{g}_{0} \equiv \boldsymbol{\sigma}\left(\boldsymbol{x}_{0}\right)
$$


Similarly

$$
\boldsymbol{y}(\mu)=\boldsymbol{\pi}\left(e^{\mu \boldsymbol{e}_{1}} \boldsymbol{g}_{0}\right) .
$$

If we now take $\pi\left(e^{\varepsilon \boldsymbol{e}_{0}} \boldsymbol{g}_{0}\right)$ as starting point and (for example) flow along $\boldsymbol{l}_{1}(\cdot)$, with parameter increase $\mu$, then by (5.5) and (5.4) we arrive at $\boldsymbol{\pi}\left(e^{\mu \boldsymbol{e}_{1}} \boldsymbol{g}_{1}\right)$, where $\boldsymbol{g}_{1}=\boldsymbol{\sigma}\left(\boldsymbol{\pi}\left(e^{\varepsilon \boldsymbol{e}_{0}} \boldsymbol{g}_{0}\right)\right)=$ $e^{\varepsilon e_{0}} \boldsymbol{g}_{0} \boldsymbol{h}$, for some $\boldsymbol{h} \in H \subset G$. Since $\boldsymbol{\pi}(\boldsymbol{a} \boldsymbol{h})=\boldsymbol{\pi}(\boldsymbol{a})$ for arbitrary $\boldsymbol{a} \in G, \boldsymbol{h} \in H$, we have

$$
\boldsymbol{\pi}\left(e^{\mu e_{1}} \boldsymbol{g}_{1}\right)=\pi\left(e^{\mu e_{1}} e^{\varepsilon e_{0}} \boldsymbol{g}_{0}\right) \text {. }
$$

It follows that

$$
S_{\Sigma}=\pi\left(D\left(e^{\varepsilon e_{0}}, e^{\mu e_{1}}\right) g_{0}\right),
$$

where $D\left(e^{\varepsilon \boldsymbol{e}_{0}}, e^{\mu \boldsymbol{e}_{1}}\right)$ is the subgroup of $G$ generated by $e^{\varepsilon \boldsymbol{e}_{0}}, e^{\mu \boldsymbol{e}_{1}}$ (denoted $D\left(\boldsymbol{a}_{0}, \boldsymbol{a}_{1}\right)$ in the Introduction). Now we study $D\left(e^{\varepsilon e_{0}}, e^{\mu e_{1}}\right)$ using the form (3.45) of the composition function, with group elements also denoted $(x, \boldsymbol{x}), x \in \mathbb{R}, \boldsymbol{x} \in \mathbb{R}^{2}$. We shall show in Proposition 2, below, that the elements of $D\left(e^{\varepsilon e_{0}}, e^{\mu e_{1}}\right)$ consist of 'integer linear combinations' of three elements (even though there are just two generators of this subgroup) - this representation allows us to discuss the discreteness of the set $S_{\Sigma}$, via (5.7). Note that from (3.45), if $(x, \boldsymbol{x}) \in G$

$$
(x, \boldsymbol{x})=\boldsymbol{\psi}((0, \boldsymbol{x}),(x, \mathbf{0})) \equiv(0, \boldsymbol{x})(x, \mathbf{0}),
$$

so that any element of $D\left(e^{\varepsilon e_{0}}, e^{\mu e_{1}}\right)$ can also be represented in this form. Also, $(0, \boldsymbol{x})$ is an element of the one-dimensional subgroup $\{(0, t \boldsymbol{x}): t \in \mathbb{R}\}$, with corresponding Lie algebra element $(0, \boldsymbol{x})$, and likewise for $(x, 0)$. Hence we may write

$$
e^{(0, \boldsymbol{x})}=(0, \boldsymbol{x}), \quad e^{(x, \boldsymbol{0})}=(x, \mathbf{0}),
$$

where on the left side of $(5.9),(0, \boldsymbol{x}) \in \boldsymbol{g}$, on the right side $(0, \boldsymbol{x}) \in G$, etc. We calculate for later use that

$$
\begin{aligned}
& (x, \boldsymbol{x})^{-1}=\left(-x,-e^{-\sigma^{\top} x} \boldsymbol{x}\right), \quad(x, \mathbf{0})^{-1}(0, \boldsymbol{x})(x, \mathbf{0})=\left(0, e^{-\sigma^{\top} x} \boldsymbol{x}\right), \\
& ((0, \boldsymbol{x}),(x, \mathbf{0})) \equiv(0, \boldsymbol{x})^{-1}(x, \mathbf{0})^{-1}(0, \boldsymbol{x})(x, \mathbf{0})=\left(0,\left(e^{-\sigma^{\top} x}-\mathbb{1}\right) \boldsymbol{x}\right) .
\end{aligned}
$$

Also, using $(5.10)_{2}$,

$$
e^{-\varepsilon \boldsymbol{e}_{0}} e^{\mu \boldsymbol{e}_{1}} e^{\varepsilon \boldsymbol{e}_{0}}=\left(0, e^{-\sigma^{\top} \varepsilon}\left(\mu \hat{\boldsymbol{e}}_{1}\right)\right), \quad \hat{\boldsymbol{e}}_{1} \equiv\left(\begin{array}{l}
1 \\
0
\end{array}\right) .
$$

Let us write $D\left(e^{\varepsilon e_{0}}, e^{\mu e_{1}}\right) \equiv D$ from now on. It is clear from (3.45) that

- if $(x, \boldsymbol{x}) \in D$, then $x=n \varepsilon$ for some $n \in \mathbb{Z}$,

- if $(0, \boldsymbol{x}),(0, \boldsymbol{y}) \in D$, then $(0, \boldsymbol{x})^{l}(0, \boldsymbol{y})^{m}=(0, l \boldsymbol{x}+m \boldsymbol{y}) \in D, l, m \in \mathbb{Z}$,

- if $(x, \boldsymbol{x}) \in D$ then $\boldsymbol{x}=\boldsymbol{\alpha} \mu$, where $\boldsymbol{\alpha}$ is independent of $\mu$.

We are interested in determining whether or not $\pi(D)$ is discrete, and it is necessary for this that $\pi\left(N^{\prime}\right)$ is discrete, whenever $N^{\prime} \subseteq D$. We notice that from (5.11) and and the last itemised statement above, $N^{\prime} \equiv(0, \mu N) \subseteq D$, where

$$
\begin{aligned}
N \equiv & \left\{\boldsymbol{p} \in \mathbb{R}^{2}: \boldsymbol{p}=l \hat{\boldsymbol{e}}_{1}+m_{1} e^{-\sigma^{\top} \varepsilon} \hat{\boldsymbol{e}}_{1}+m_{2} e^{-2 \sigma^{\top} \varepsilon} \hat{\boldsymbol{e}}_{1}\right. \\
& \left.+\cdots+m_{n} e^{-n \sigma^{\top} \varepsilon} \hat{\boldsymbol{e}}_{1}, l, m_{1}, \ldots, m_{n}, n \in \mathbb{Z}\right\} .
\end{aligned}
$$

Notice that for any $\boldsymbol{p}, \boldsymbol{q} \in N, \alpha \boldsymbol{p}+\beta \boldsymbol{q} \in N, e^{-\sigma^{\top} \varepsilon} \boldsymbol{p} \in N, \alpha, \beta \in \mathbb{Z}$. 
Remark Recall from Sect. 4 that two of the projections that occur have the form $\pi(z, x, y)=$ $(x, y)$ (the same projection occurs both when $\sigma=\left(\begin{array}{cc}1 & 0 \\ 0 & -1\end{array}\right)$ and when $\sigma=\left(\begin{array}{cc}0 & +1 \\ -1 & 0\end{array}\right)$ ). So in these two cases $\pi\left(N^{\prime}\right)=N$. Therefore we consider these two cases first, and enquire in each case if $\boldsymbol{\pi}\left(N^{\prime}\right)=N$ is discrete.

(i) Suppose that $\sigma=\left(\begin{array}{cc}0 & 1 \\ -1 & 0\end{array}\right)$ with Lie algebra basis (from (3.18)) such that $\left[\boldsymbol{l}_{1}, \boldsymbol{l}_{2}\right]=\mathbf{0}$, $\left[\boldsymbol{l}_{0}, \boldsymbol{l}_{1}\right]=\boldsymbol{l}_{2},\left[\boldsymbol{l}_{0}, \boldsymbol{l}_{2}\right]=-\boldsymbol{l}_{1}$. Then (1.20) holds with $\boldsymbol{l}_{2}=\left[\boldsymbol{l}_{0}, \boldsymbol{l}_{1}\right], \beta=-1$, other constants zero. Set

$$
e^{\sigma^{\top} \varepsilon} \equiv R(\varepsilon) \equiv\left(\begin{array}{cc}
\cos \varepsilon & -\sin \varepsilon \\
\sin \varepsilon & \cos \varepsilon
\end{array}\right)
$$

$N$ is to be discrete, and from the remark following (5.12) it follows that $N$ is invariant under rotation through $\varepsilon$. Let $\boldsymbol{p}, \boldsymbol{q} \in N$ be two points of $N$ such that the distance between them (as points in $\mathbb{R}^{2}$ ) is a minimum, then by the standard crystallography argument (in $\mathbb{R}^{2}$ ), using the remark following (5.12), one can obtain a contradiction unless

$$
\frac{\varepsilon}{2 \pi}=\frac{1}{p}, \quad p=1,2,3,4,6 .
$$

Hence (5.14) is necessary if $D$ is to be discrete. According to results in Sect. 3, for each such choice of $\varepsilon$, there is a matrix $\gamma \in \mathrm{SL}_{2}(\mathbb{Z})$ such that

$$
e^{\sigma^{\top} \varepsilon}=P \gamma P^{-1}, \quad P \in \mathrm{GL}_{2}(\mathbb{R}),
$$

and the corresponding matrices $\gamma$ as given in Sect. 3. Hence if $(n \varepsilon, \boldsymbol{x}),(m \varepsilon, \boldsymbol{y}) \in D$ we have

$$
\begin{aligned}
(n \varepsilon, \boldsymbol{x})(m \varepsilon, \boldsymbol{y}) & =\left((n+m) \varepsilon, \boldsymbol{x}+e^{n \sigma^{\top} \varepsilon} \boldsymbol{y}\right) \\
& =\left((n+m) \varepsilon, \boldsymbol{x}+P \gamma^{n} P^{-1} \boldsymbol{y}\right) \\
& =\left((n+m) \varepsilon, P\left(P^{-1} \boldsymbol{x}+\gamma^{n} P^{-1} \boldsymbol{y}\right)\right) .
\end{aligned}
$$

Proposition 2 Suppose that $\sigma^{\top} \varepsilon$ is such that (5.15) holds with $\gamma \in S L_{2}(\mathbb{Z})$, then all elements of $D$ can be represented as integer linear combinations of $\varepsilon \boldsymbol{e}_{0},\left(0, \mu \hat{\boldsymbol{e}}_{1}\right),\left(0, e^{\sigma^{\top} \varepsilon}\left(\mu \hat{\boldsymbol{e}}_{1}\right)\right)$, and all such linear combinations correspond to elements of $D$.

Proof We only have to show that

$$
(0, \boldsymbol{x}) \in D \text { if and only if } \boldsymbol{x}=\mu\left(l \hat{\boldsymbol{e}}_{1}+m e^{\sigma^{\top} \varepsilon} \hat{\boldsymbol{e}}_{1}\right), \quad l, m \in \mathbb{Z},
$$

for the following reason. Recall that $(x, 0) \in D$ if and only if $x=n \varepsilon$ for some $n \in \mathbb{Z}$. Then if $(x, \boldsymbol{x}) \in G$ is a linear combination of the terms given in the statement of the proposition, we have that $(x, \boldsymbol{x})=(0, \boldsymbol{x})(x, 0) \in D$ using the fact recalled and (5.17). Conversely, if $(x, \boldsymbol{x}) \in D$ we have that $x=n \varepsilon, n \in \mathbb{Z}$, so $(x, 0) \in D$. But then $(0, \boldsymbol{x})=(x, \boldsymbol{x})(x, 0)^{-1} \in D$, so by (5.17), $\boldsymbol{x}$ is an integer linear combination of $\mu \hat{\boldsymbol{e}}_{1}, e^{\sigma^{\top} \varepsilon}\left(\mu \hat{\boldsymbol{e}}_{1}\right)$.

Note that

$$
(0, \boldsymbol{x})=\left(0, l\left(\mu \hat{\boldsymbol{e}}_{1}\right)+m e^{\sigma^{\top} \varepsilon}\left(\mu \hat{\boldsymbol{e}}_{1}\right)\right)=\left(0, \mu \hat{\boldsymbol{e}}_{1}\right)^{l}\left(0, e^{\sigma^{\top} \varepsilon}\left(\mu \hat{\boldsymbol{e}}_{1}\right)\right)^{m} \in D,
$$


so we just have to prove the 'only if' implication in (5.17). So let $\boldsymbol{g} \equiv(x, \boldsymbol{x}) \in D$. Then $\boldsymbol{g}$ can be written as a product of the generators $e^{\varepsilon e_{0}}, e^{\mu e_{1}}$ and their inverses (called a 'word' in the generators). Let $n$ be the minimum length of any such (i.e., $=g$ ) word in the generators. Clearly the desired implication holds if $n=1$. To prove the result by induction on $n$ it suffices to show that $\boldsymbol{f}\left(k \varepsilon, l\left(\mu \hat{\boldsymbol{e}}_{1}\right)+m e^{\sigma^{\top} \varepsilon}\left(\mu \hat{\boldsymbol{e}}_{1}\right)\right)$ has the required form when $\boldsymbol{f}$ is one of the generators or their inverses, $k, l, m \in \mathbb{Z}$. It turns out that the only non-trivial task is to rearrange

$$
\left(\varepsilon \boldsymbol{e}_{0}\right)\left(k \varepsilon, l\left(\mu \hat{\boldsymbol{e}}_{1}\right)+m e^{\sigma^{\top} \varepsilon}\left(\mu \hat{\boldsymbol{e}}_{1}\right)\right)=\left((k+1) \varepsilon, e^{\sigma^{\top} \varepsilon}\left(\lambda\left(\mu \hat{\boldsymbol{e}}_{1}\right)+m e^{\sigma^{\top} \varepsilon}\left(\mu \hat{\boldsymbol{e}}_{1}\right)\right)\right) .
$$

But $\left(e^{\sigma^{\top} \varepsilon}\right)^{2}=\left(\operatorname{tr} e^{\sigma^{\top} \varepsilon}\right) e^{\sigma^{\top} \varepsilon}-\mathbb{1}=(\operatorname{tr} \gamma) e^{\sigma^{\top} \varepsilon}-\mathbb{1}$, by (5.15), and $\operatorname{tr} \gamma \in \mathbb{Z}$. This proves the result.

\section{Remark}

The proposition holds independently of the choice of $\hat{\boldsymbol{e}}_{1}=\left(\begin{array}{l}a \\ b\end{array}\right) \neq \mathbf{0}$.

It follows that

$$
\pi(D)=\left\{l\left(\mu \hat{\boldsymbol{e}}_{1}\right)+m e^{\sigma^{\top} \varepsilon}\left(\mu \hat{\boldsymbol{e}}_{1}\right): l, m \in \mathbb{Z}\right\},
$$

where $\varepsilon$ is such that (5.14) holds, so $\pi(D)$ is a two-dimensional lattice, with integral basis $\mu \hat{\boldsymbol{e}}_{1}, e^{\sigma^{\top} \varepsilon}\left(\mu \hat{\boldsymbol{e}}_{1}\right)$, for this choice of isotopy group/projection/group action. Note that the fundamental cell of the lattice can be arbitrarily small.

(ii) Now suppose that $\sigma=\left(\begin{array}{cc}1 & 0 \\ 0 & -1\end{array}\right), \pi(z, x, y)=(x, y)$. The Lie algebra basis such that $\left[\boldsymbol{l}_{0}, \boldsymbol{l}_{1}\right]=\boldsymbol{l}_{1},\left[\boldsymbol{l}_{0}, \boldsymbol{l}_{2}\right]=-\boldsymbol{l}_{2},\left[\boldsymbol{l}_{1}, \boldsymbol{l}_{2}\right]=\mathbf{0}$ (from (3.18)) is of the form (1.20). So we take $\tilde{\boldsymbol{l}}_{0} \equiv \boldsymbol{l}_{0}, \tilde{\boldsymbol{l}}_{1}=\boldsymbol{l}_{1}+\boldsymbol{l}_{2}, \tilde{\boldsymbol{l}}_{2}=\boldsymbol{l}_{1}-\boldsymbol{l}_{2}$, to get $\left[\tilde{\boldsymbol{l}}_{0}, \tilde{\boldsymbol{l}}_{1}\right]=\tilde{\boldsymbol{l}}_{2},\left[\tilde{\boldsymbol{l}}_{0}, \tilde{\boldsymbol{l}}_{2}\right]=\tilde{\boldsymbol{l}}_{1},\left[\tilde{\boldsymbol{l}}_{1}, \tilde{\boldsymbol{l}}_{2}\right]=\mathbf{0}$, which has the form (1.20) with $\tilde{\boldsymbol{l}}_{2}=\left[\tilde{\boldsymbol{l}}_{0}, \tilde{\boldsymbol{l}}_{1}\right]$, with $\beta=1$, other constants zero. So here we are interested in the subgroup generated by $e^{\varepsilon e_{0}}, e^{\mu\left(\boldsymbol{e}_{1}+\boldsymbol{e}_{2}\right)}$-we continue to denote the subgroup by $D$. Note that $e^{\mu\left(\boldsymbol{e}_{1}+\boldsymbol{e}_{2}\right)}=e^{\mu \boldsymbol{e}_{1}} e^{\mu \boldsymbol{e}_{2}}=\left(0, \mu\left(\hat{\boldsymbol{e}}_{1}+\hat{\boldsymbol{e}}_{2}\right)\right)$, where (3.45) holds.

We shall assume, in this case, that $\varepsilon$ is such that (5.15) holds, so

$$
\operatorname{tr} e^{\sigma^{\top} \varepsilon}=e^{\varepsilon}+e^{-\varepsilon}=\operatorname{tr} \gamma \in \mathbb{Z} .
$$

Given this assumption, from Proposition 2 (and the remark that follows it)

$$
\begin{aligned}
\pi(D) & =\left\{\mu\left(l\left(\begin{array}{l}
1 \\
1
\end{array}\right)+m\left(\begin{array}{cc}
e^{\varepsilon} & 0 \\
0 & e^{-\varepsilon}
\end{array}\right)\left(\begin{array}{l}
1 \\
1
\end{array}\right)\right): l, m \in \mathbb{Z}\right\} \\
& =\left\{\mu\left(l+m e^{\varepsilon}, l+m e^{-\varepsilon}\right)^{\top}: l, m \in \mathbb{Z}\right\} .
\end{aligned}
$$

This set is not discrete, for if it were then $e^{\varepsilon}$ would be rational, so $e^{\varepsilon}=p / q, p, q \in \mathbb{Z}$ relatively prime. Then (5.21) gives that $p^{2} / q=n p-q$ for some integer $n$, which implies that $q$ divides $p$, so $q=1$ and $e^{\varepsilon}=p$, which is impossible.

It remains to consider whether or not $\pi(D)$ is discrete in two cases:

(iii) $\sigma=\left(\begin{array}{cc}0 & 1 \\ -1 & 0\end{array}\right), \pi(z, x, y)=(z, y \cos z-x \sin z)$, generators $e^{\sigma e_{0}}, e^{\mu e_{1}}$;

(iv) $\sigma=\left(\begin{array}{cc}1 & 0 \\ 0 & -1\end{array}\right), \pi(z, x, y)=\left(z, x e^{-z}-y e^{z}\right)$, generators $e^{\varepsilon e_{0}}, e^{\mu\left(e_{1}+e_{2}\right)}$. 
We shall assume that (5.14), (5.15) hold in case (iii), and that (5.15) holds in case (iv), so that Proposition 2 holds in each case, to simplify the calculations. (We have no results in the case that (5.15) does not hold.)

In case (iii), elements of $D$ are integer linear combinations of $\varepsilon \boldsymbol{e}_{0},\left(0, \mu \hat{\boldsymbol{e}}_{1}\right),\left(0, R(\varepsilon) \mu \hat{\boldsymbol{e}}_{1}\right)$, and one calculates that

$$
\pi(D)=\{(l \varepsilon, \mu(n \sin \varepsilon \cos l \varepsilon-(m+n \cos \varepsilon) \sin l \varepsilon)): l, m, n \in \mathbb{Z}\} \equiv \bigcup_{l \in \mathbb{Z}}\left(l \varepsilon, \mu S_{l}\right),
$$

where we define

$$
S_{l} \equiv\{-m \sin l \varepsilon+n \sin (l-1) \varepsilon: m, n \in \mathbb{Z}\}
$$

We shall show that

$$
S_{l}=S_{1}=S_{0}=\{m \sin \varepsilon: m \in \mathbb{Z}\}, \quad l \in \mathbb{Z} .
$$

It is clear that $S_{1}=S_{0}$. Recall that $\varepsilon=\frac{2 \pi}{p}, p=1,2,3,4$ or 6 , and notice from the definition that

$$
S_{l}=S_{l+p}=S_{p+1-l}, \quad l \in \mathbb{Z} .
$$

We prove (5.25) for each value of $p$ in turn, beginning with $p=2$-then $S_{0}=S_{1}$ and (5.26) gives the result. For $p=3$, we only have to prove that $S_{2}=S_{1}$ (from (5.26)), but this is so because $\sin 4 \pi / 3=-\sin 2 \pi / 3$. The other cases are similar. Hence

$$
\pi(D)=\{(l \varepsilon, \mu m \sin \varepsilon): l, m \in \mathbb{Z}\},
$$

so if $p=1,2$ this is a one-dimensional lattice, and if $p=3,4,6$ it is a two-dimensional lattice.

In case (iv), elements of $D$ are integer linear combinations of $\varepsilon \boldsymbol{e}_{0},\left(0, \mu\left(\hat{\boldsymbol{e}}_{1}+\hat{\boldsymbol{e}}_{2}\right)\right)$, $\left(0, \mu\left(\begin{array}{cc}e^{\varepsilon} & 0 \\ 0 & e^{-\varepsilon}\end{array}\right)\left(\begin{array}{l}1 \\ 1\end{array}\right)\right)$ and one calculates from $\pi(z, x, y)=\left(z, x e^{-z}-y e^{z}\right)$ that

$$
\pi(D)=\bigcup_{l \in \mathbb{Z}}\left(l_{\varepsilon}, \mu S_{l}\right)
$$

where we define

$$
S_{l}=\left\{m\left(e^{l \varepsilon}-e^{-l \varepsilon}\right)+n\left(e^{(l-1) \varepsilon}-e^{-(l-1) \varepsilon}\right): m, n \in \mathbb{Z}\right\} .
$$

Recall that $e^{\varepsilon}+e^{-\varepsilon} \in \mathbb{Z}$, by assumption. We notice that, for $k \in \mathbb{Z}$

$$
\frac{\sinh k \varepsilon}{\sinh \varepsilon}=e^{(k-1) \varepsilon}+e^{(k-3) \varepsilon}+\cdots+e^{-(k-3) \varepsilon}+e^{-(k-1) \varepsilon},
$$

which is a sum of terms of the form $e^{r \varepsilon}+e^{-r \varepsilon}$, or 1 . But

$$
\left(e^{(r+1) \varepsilon}+e^{-(r+1) \varepsilon}\right)=\left(e^{r \varepsilon}+e^{-r \varepsilon}\right)\left(e^{\varepsilon}+e^{-\varepsilon}\right)-\left(e^{(r-1) \varepsilon}+e^{-(r-1) \varepsilon}\right),
$$

so by induction we see that $e^{r \varepsilon}+e^{-r \varepsilon} \in \mathbb{Z}, r>0, r$ an integer. Therefore we define

$$
a_{k}=\frac{\sinh k \varepsilon}{\sinh \varepsilon} \in \mathbb{Z}
$$


$a_{k}$ is a polynomial of degree $(k-1)$ in the variable $\left(e^{\varepsilon}+e^{-\varepsilon}\right)$ (Chebyshev polynomial of the second kind). One checks that

$$
a_{k+1}=p a_{k}-a_{k-1}, \quad a_{0}=0, a_{1}=1,
$$

where $p \equiv e^{\varepsilon}+e^{-\varepsilon} \in \mathbb{Z}$.

Now $S_{l}$ can be written as

$$
S_{l}=\left(e^{\varepsilon}-e^{-\varepsilon}\right)\left\{m a_{l}+n a_{l-1}: m, n \in \mathbb{Z}\right\} .
$$

By (5.34) this can be rearranged as

$$
\begin{aligned}
S_{l} & =\left(e^{\varepsilon}-e^{-\varepsilon}\right)\left\{(n+m p) a_{l-1}-m a_{l-2}: m, n \in \mathbb{Z}\right\} \\
& =\left(e^{\varepsilon}-e^{-\varepsilon}\right)\left\{m^{\prime} a_{l-1}+n^{\prime} a_{l-2}: m^{\prime}, n^{\prime} \in \mathbb{Z}\right\} \\
& =S_{l-1} .
\end{aligned}
$$

It follows that

$$
S_{l}=S_{1}=\left(e^{\varepsilon}-e^{-\varepsilon}\right) \mathbb{Z} .
$$

Hence from (5.29), $\boldsymbol{\pi}(D)$ is a lattice with basis $(\varepsilon, \mathbf{0}), \mu\left(e^{\varepsilon}-e^{-\varepsilon}\right)\left(0, \hat{\boldsymbol{e}}_{1}+\hat{\boldsymbol{e}}_{2}\right)$.

Incidentally notice that

$$
\left(\begin{array}{c}
e^{l \varepsilon} \\
e^{-l \varepsilon}
\end{array}\right)=a_{l}\left(\begin{array}{c}
e^{\varepsilon} \\
e^{-\varepsilon}
\end{array}\right)-a_{l-1}\left(\begin{array}{l}
1 \\
1
\end{array}\right),
$$

so that $\left(\begin{array}{c}e^{l \varepsilon} \\ e^{-l \varepsilon}\end{array}\right)$ is an element of the sublattice $V$ of $D$ with basis $\left(\begin{array}{l}1 \\ 1\end{array}\right),\left(\begin{array}{c}e^{\varepsilon} \\ e^{-\varepsilon}\end{array}\right)$ (omitting the first component). The relevant projection mapping represents cosets in terms of lines parallel to $\left(\begin{array}{c}e^{l \varepsilon} \\ e^{-l \varepsilon}\end{array}\right)$ in planes $z=l \varepsilon$, so from (5.37) these lines are rational directions in $V$. The fact that $S_{l}$ has the form (5.36) is thus a direct reflection of the fact that the distance between adjacent parallel rational lines in $V$ is constant.

\section{Conclusion}

We have discussed both continuous and discrete structures associated with crystals that have non-constant dislocation density and unimodular solvable group structure, above. There are some more cases that need to be dealt with before we can progress to the next stage of this programme with a degree of understanding sufficient to address variational problems (say) which model defective crystals with strain energy densities of the type described in Sect. 1. Specifically, we need to consider the case where the relevant Lie group is simple. In particular, the group SU(2) appears-the topology of the corresponding homogeneous space is non-trivial (it has curvature), and the apparatus of Sect. 2 is not quite refined enough to discuss this particular case. (The theory of fibre bundles is the extra ingredient required.)

Once the simple case is dealt with, the next task to consider the 'symmetries' of the discrete structures so obtained, and the main issue then is the following: the symmetries of the discrete structures are automorphisms of the corresponding discrete groups-there is no general reason (apparently) why these automorphisms should extend to automorphisms of the ambient (continuous) Lie group. Now the automorphisms of the continuous group, 
in continuum mechanics terms, can be thought of as elastic deformations-so, rephrasing, the issue is whether or not the discrete symmetries can be embedded (perhaps uniquely) in (smooth) elastic deformations. But if these symmetries may indeed be so extended, then they may be taken as 'material symmetries' for strain energy functions which model defective crystals, in generalization of classical work which uses the crystal classes as material symmetries of strain energy densities for perfect crystals.

Finally we remark that the theory of fibre bundles has a well established and prominent position in modern theoretical physics - it leads (for example) to the existence of localized disturbances which satisfy field equations deriving from certain variational problems. So the emergence of this theory in the context of defective crystals is exciting, since the idea that defects have a similar status, as smooth localized disturbances in a continuum mechanics setting, is a long-held ideal (by some).

Acknowledgements This work was supported by the Engineering and Physical Sciences Research Council [grant number EP/M024202/1], we are very grateful to the EPSRC for the support.

Publisher's Note Springer Nature remains neutral with regard to jurisdictional claims in published maps and institutional affiliations.

Open Access This article is distributed under the terms of the Creative Commons Attribution 4.0 International License (http://creativecommons.org/licenses/by/4.0/), which permits unrestricted use, distribution, and reproduction in any medium, provided you give appropriate credit to the original author(s) and the source, provide a link to the Creative Commons license, and indicate if changes were made.

\section{References}

1. Parry, G.P., Zyskin, M.: Geometrical structure of two-dimensional crystals with non-constant dislocation density. J. Elast. 127, 249-268 (2017). https://doi.org/10.1007/s10659-016-9612-3

2. Davini, C.: A proposal for a continuum theory of defective crystals. Arch. Ration. Mech. Anal. 96, 295317 (1986). https://doi.org/10.1007/BF00251800

3. Davini, C., Parry, G.P.: On defect-preserving deformations in crystals. Int. J. Plast. 5, 337-369 (1989). https://doi.org/10.1016/0749-6419(89)90022-3

4. Davini, C., Parry, G.P.: A complete list of invariants for defective crystals. Proc. R. Soc. Lond. A 432, 341-365 (1991); Erratum: Proc. R. Soc. Lond. A 434, 735 (1991). https://doi.org/10.1098/ rspa.1991.0125

5. Olver, P.J.: Equivalence, Invariants and Symmetry. Cambridge University Press, Cambridge (1996)

6. Parry, G.P., Šilhavý, M.: Elastic invariants in the theory of defective crystals. Proc. R. Soc. Lond. A 455, 4333-4346 (1999). https://doi.org/10.1098/rspa.1999.0503

7. Elżanowski, M., Preston, S.: On continuously defective elastic crystals. Miskolc Math. Notes 14(2), 659-670 (2013)

8. Bourbaki, N.: Elements of Mathematics, Part 2: General Topology. Hermann, Paris (1996). https:// doi.org/10.4007/annals.2003.157.575

9. Olver, P.J.: Applications of Lie Groups to Differential Equations, 2nd edn. Graduate Texts in Mathematics, vol. 107. Springer, Berlin (1993)

10. Palais, R.S.: A Global Formulation of the Lie Theory of Transformation Groups. Mem. Amer. Math. Soc., vol. 22 (1957)

11. Bianchi, L.: Lezioni sulla teoria dei gruppi continui finiti di trasformazioni. Enrico Spoerri, Editore, Pisa (1918)

12. Jacobsen, N.: Lie Algebras. Dover Publications, New York (1979)

13. Auslander, L., Green, L., Hahn, F.: Flows on Homogeneous Spaces. Annals of Mathematics Studies, vol. 53. Princeton University Press, Princeton (1963)

14. Fonseca, I., Parry, G.P.: Equilibrium configurations of defective crystals. Arch. Ration. Mech. Anal. 120, 245-283 (1992). https://doi.org/10.1007/BF00375027

15. Pontryagin, L.S.: Topological Groups, 2nd edn. Gordon and Breach, New York/London/Paris (1955)

16. Komrakov, B., Churyamov, A., Doubrov, B.: Two-dimensional homogeneous spaces. Preprint Series 17-Matematisk institutt, Universitetet i Oslo (1993) 
17. Ha, K.Y., Lee, J.B.: Left invariant metrics and curvatures on simply connected three-dimensional Lie groups. Math. Nachr. 282(6), 868-898 (2009). https://doi.org/10.1002/mana.200610777

18. Nicks, R., Parry, G.P.: On symmetries of crystals with defects related to a class of solvable groups $\left(S_{1}\right)$. Math. Mech. Solids 17, 631-651 (2011). https://doi.org/10.1177/1081286511427485

19. Rossmann, W.: Lie Groups, an Introduction Through Linear Groups. Oxford Graduate Texts in Mathematics, vol. 5. Oxford University Press, Oxford (2006) 